\title{
Co-evolution of construction waste recycling industrial chain based on Lotka-Volterra model
}

\author{
Yanqing Xiao ${ }^{1,2}$, Jingkuang Liu ${ }^{2 *}$, Yongshi Pang ${ }^{2}$ \\ ${ }^{1}$ Guangzhou Municipal Construction Group Co., Ltd., \\ Guangzhou, 5100305 \\ China \\ ${ }^{2}$ Guangzhou University, \\ Guangzhou, 510006,China, \\ E-mail: somliujingkuang@gzhu.edu.cn
}

Received: February 2, 2021. Revised: July 8, 2021. Accepted: August 3, 2021. Published: August 5, 2021.

\begin{abstract}
Under the green building policy, the recycling of construction waste has become an important issue. However, many obstructions in the industrial chain of construction waste recycling, slow integration of production elements in the chain, and inefficient resource allocation hinder the development of the construction waste recycling industry. In this study, a co-evolution model of the industrial chain of construction waste recycling is constructed based on the Lotka-Volterra model, and the Jacobian matrix is used for stability analysis. Finally, a numerical simulation is performed. The simulation results indicate that: (1) There is a high product correlation between upstream and downstream enterprises in the industrial chain of construction waste recycling; (2) The conversion coefficient of supply and demand of upstream and downstream enterprises has a higher impact on and is more sensitive to the evolution of this industrial chain; (3) The co-evolution of upstream and downstream enterprises promotes the added value of products and maximizes the overall benefits of the industrial chain, which provides reference value and theoretical basis for the development of the industrial chain of construction waste recycling.
\end{abstract}

Keywords-Construction waste, Lotka-Volterra, coevolution, recycling, industrial chain.

\section{INTRODUCTION}

$\mathrm{C}$ OnSTRUCTION waste recycling has been the focus of experts and government departments around the world. However, continuous deepening of urbanization construction in China has also resulted in a large amount of construction waste, a part of which is simply landfilled or inadequately disposed, resulting in waste of resources and land, and seriously affecting the surrounding environment of the city [1]. According to statistics, the amount of construction waste in China accounts for 30\%$40 \%$ of the total urban waste [2]. Although China has progressed in reducing construction waste, the efficiency of construction waste recycling is relatively low, and the utilization rate of resources is less than $5 \%$. In contrast, in accordance with the European Revised Waste Framework Directive 2008/98/EC, European countries should recover at least $70 \%$ of nonhazardous construction and demolition waste by 2020 [3] - [5]. Thus, China exhibits a discrepancy compared to developed countries regarding the recycling of construction waste.

The industrial chain of construction waste recycling refers to an integration including value, enterprise, supply, and space chains, which are mainly composed of 
construction contractor enterprises, construction waste recycling enterprises, and recycled product dealers, with construction waste and recycled products as series products, guided by market demand [6]. Considering the industrial chain of construction waste recycling in China, the industrial chain ecosystem is shown in Fig. 1.

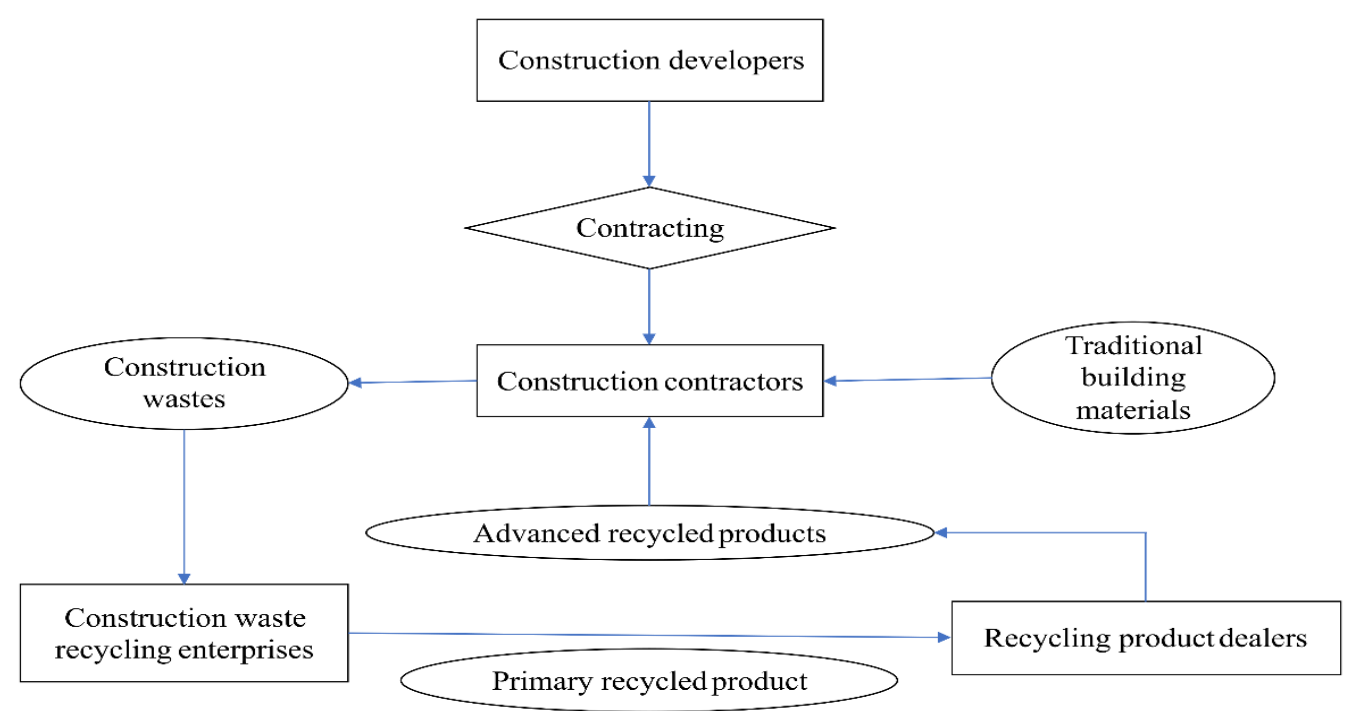

Fig. 1 Ecosystem of the construction waste recycling industrial chain

The construction developers contract the project to the construction contractors, who produce construction wastes during the implementation of the project. The construction contractors transport construction wastes to the recycling enterprises, which transform the wastes into construction material products (primary recycled products) through classification, processing, and other processes. The construction waste recycling enterprises then transport the recycled products to the recycled product dealers to produce advanced recycled products that will be sold to the construction contractors for the development of a project. This industrial chain reflects the process of sharing, interaction, integration, and distribution of production factors such as capital, knowledge, technology, information, and resources, regardless of the type of enterprise, whether it is an internal enterprise such as construction waste recycling enterprises, developers, building material dealers, and construction contractors, or the interaction between upstream and downstream enterprises in the industrial chain (including circulation and information transmission between upstream and downstream enterprises, such as construction waste and recycled products) [7] [8]. However, there are many obstructions in China's construction waste recycling industry chain, such as slow integration of production elements in the chain and inefficient resource allocation, which hinder the development of the construction waste recycling industry [9]. This is mainly due to: (1) Lack of systematic resource management thinking, which results in the diversity and complexity of participants, dynamics and competitiveness of the market environment, inadequate macro-control efforts of the government, and untimely management of industrial chain problems in the process of construction waste recycling management. An example is the " $12 \cdot 20$ " particularly serious landslide accident at a residue acceptance site of the Guangming New Area in Shenzhen, which was mainly caused by the upstream industry in the industrial chain of urban construction waste [10]. In this case, Shenzhen Municipal Government could only compensate the loss after the incident by attaching considerable importance to the recycling of construction waste, promulgating corresponding support and subsidy 
policies, promoting the classification of the sources, and improving the recycling efficiency of construction waste; (2) Lack of regular understanding of the coordinated development of the industrial chain. The development and perfection of the industrial chain of construction waste recycling is regular: The government should support the construction waste recycling industry differently at each stage and under diverse economic development conditions, as the main body of the ecosystem of the industrial chain reacts differently. However, the laws of internal development and evolution have not been understood by local governments, and the economic incentive policies formulated during the growth of industrial chains have little effect, combined with insufficient support and insufficient power to support industrial development, which hinders the development and improvement of the industrial chain of construction waste recycling. Based on the aforementioned information, in this study, the relationship between the participants in the industrial chain of construction waste recycling is studied, the co-evolution law of the industrial chain of construction waste is discussed, and strategies and suggestions for promoting the development of this chain are proposed.

\section{LITERATURE REVIEW}

A mature industrial chain of construction waste recycling and its smooth operation and development have significant importance to solve the problem of construction waste disposal. In recent years, researchers have focused on the industrial chain of construction waste recycling [11]. Based on the diamond model, Hu et al. analyzed the effects of production factors, demand conditions, enterprise strategy, related industries, development opportunities, and government actions on the development of the construction waste recycling industry [12]. Gao and Xie based on the development of an industrial chain structure model of construction waste recycling, cited as implementation strategies the promotion of the development of recycling enterprises, maintenance of a stable supply of raw materials in the industrial chain, and improvement of the market competitiveness of recycling products [13]. Liu et al. constructed a green building industry chain model from the perspective of recycling economy and analyzed the interaction between the government and builders in this chain [14]. They suggested the strengthening of the management of recycling parts in the chain, acceleration of the complex development of the green building industry chain, establishment of a green building industry alliance, and formulation and improvement of relevant incentive policies by the government. Kucukvar et al. analyzed the optimal strategy selection of recycling, landfilling, and incineration strategies in the entire economic supply chain under different evaluation indices by constructing a mixed life cycle assessment model based on economic input-output [15]. Li et al. discussed the logistics network design scheme of the construction waste reduction system under the condition of site treatment capacity availability, and constructed a logistics network model of the reduction system [16]. Wu et al. believed that the cross-regional flow of construction waste can affect the quantity of waste in the regional waste management system and produce economic, social, and environmental impacts to the system [17].

Lotka and Volterra established the predator-prey system and the competitive system models, respectively and Odum extended these models to the reciprocal system, which is known as the Lotka-Volterra model [18] - [20]. Nowadays, this model is widely applied by many researchers in the fields of inter-industry coupling relationships, energy industry competition, futures index competition, relations between majority and minority shareholders in a shareholder population, economic growth, and energy consumption relationships. For example, Zhou and Wang used the Lotka-Volterra model to measure the symbiotic relationship and symbiotic coordination degree of industrial economy and industrial ecology in 30 provinces and regions of China, respectively, and classified the coordination types of industrial ecoeconomic systems according to symbiotic relationships and development levels, analyzing influencing factors [21]. Yang and $\mathrm{Hu}$ used the Lotka-Volterra model to measure the degree of coordination of the eco-economic system in ethnic minority areas from 2011 to 2015, and applied a neural network model to analyze the main factors affecting 
the degree of coordination [22]. Szolnoki et al. utilized the Lotka-Volterra model to study the coordination and competition among biological populations, and used the RP model and Ginzburg-Landau theory to build a large ecological system [23]. Lauro et al. used the LotkaVolterra model to construct the coordination of microorganisms in a lake, highlighting the coordination relationship among populations, and contributing to the study of the ecological environment system in the lake [24]. Bao et al. used the Lotka-Volterra model to study the degree of coordination of population dynamics [25]. In the current research on industrial chains, the Lotka-Volterra model is a more advanced and effective method for dynamic research on synergy, competition, and symbiosis between upstream and downstream of the industrial chain. Thus, the Lotka-Volterra model is first introduced to study the relationship between upstream and downstream of the construction waste recycling industry chain. Then, the evolution process of the upstream and downstream enterprises in the construction waste recycling industrial chain is analyzed from an ecological perspective, a coevolution model of this chain is constructed, and strategies and suggestions to promote the development of the industrial chain of construction waste recycling are drawn using a numerical simulation.

\section{Methodology}

Interdependence and cooperation between organisms and between organisms and the environment are the basis for survival, and co-evolution occurs every moment in the biological field. During the operation of the industrial chain of construction waste recycling, the synergy between the upstream and downstream of the industrial chain reflects the process in which these parts correlate, benefit, and restrict each other. The similarities between organisms and enterprises suggest a co-evolution process between upstream and downstream enterprises in the development and perfection of the industrial chain of construction waste recycling, as they interact and develop together in the system and constantly adjust in the process of co-evolution to maximize the value of the industrial chain. Therefore, in this study, a co-evolution model on the industrial chain of construction waste recycling is constructed by referring to the collaborative evolution theory in ecology and the Lotka-Volterra model. First, the basic hypotheses of the Lotka-Volterra model are discussed. In sequence, the coevolution model of the construction waste industry chain is constructed, and numerical simulation and analysis are performed. Finally, case simulation and modeling are performed to draw conclusions and suggestions.

\section{A. Basic hypotheses}

The following reasonable hypotheses are made according to the actual situation and many studies before building a co-evolution model for the industrial chain of construction waste recycling [26] - [32]:

1) To simplify, the impact of minor enterprises, such as subcontractors, is ignored. The recycling industry chain analyzed consists of construction contractors, construction waste recycling enterprises, and recycled product dealers.

2) The delay in the production and transportation of construction waste recycling enterprises is not considered, and the supply and demand of construction waste and recycled products are assumed to be uniform.

3) Regardless of the impact of the type of project, it is assumed that the total amount of building materials (including recycled products and traditional building materials) equals the amount of construction waste generated + the amount used in the building.

\section{B. Definition of parameters and related decision variables}

Based on the basic hypotheses of the model, the parameters and definitions of the co-evolution model are shown in Table I. 
Table I. Parameters and definitions of the co-evolution model

\begin{tabular}{|c|c|c|}
\hline Parameters & Definitions & Value range \\
\hline$\beta_{1}$ & Natural growth rate of construction contractors & $\beta_{1} \in[-1,1]$ \\
\hline$\beta_{2}$ & Natural growth rate of construction waste recycling enterprises & $\beta_{2} \in[-1,1]$ \\
\hline$\beta_{3}$ & $\begin{array}{l}\text { Natural growth rate of dealers of recycled construction waste } \\
\text { products }\end{array}$ & $\beta_{3} \in[-1,1]$ \\
\hline$\varepsilon_{1}$ & $\begin{array}{l}\text { Conversion coefficient of demand for construction waste of the } \\
\text { downstream construction waste recycling enterprises } \\
\text { (considering the upstream and downstream industrial chain } \\
\text { between construction contractors and construction waste } \\
\text { recycling enterprises) }\end{array}$ & $\varepsilon_{1}>0$ \\
\hline$\varepsilon_{2}$ & $\begin{array}{l}\text { Conversion coefficient of demand for recycled products of the } \\
\text { downstream recycled products dealers (considering the } \\
\text { upstream and downstream industrial chain between construction } \\
\text { waste recycling enterprises and recycled products dealers) }\end{array}$ & $\varepsilon_{2}>0$ \\
\hline$\tau_{1}$ & $\begin{array}{l}\text { Conversion coefficient of supply of construction waste from } \\
\text { upstream construction contractors (considering the upstream and } \\
\text { downstream industrial chain between construction contractors } \\
\text { and construction waste recycling enterprises) }\end{array}$ & $\tau_{1}>0$ \\
\hline$\tau_{2}$ & $\begin{array}{l}\text { Conversion coefficient of supply of recycled products of the } \\
\text { upstream construction waste recycling enterprises (considering } \\
\text { the upstream and downstream industrial chain between } \\
\text { construction waste recycling enterprises and recycled products } \\
\text { dealers) }\end{array}$ & $\tau_{2}>0$ \\
\hline$D$ & $\begin{array}{l}\text { Maximum amount of construction waste (including waste from } \\
\text { recycled products and traditional building materials, as well as } \\
\text { waste such as residues) generated in the upstream and } \\
\text { downstream industrial chain between the construction } \\
\text { contractors and the construction waste recycling enterprises, } \\
\text { with sufficient resources for the upstream construction } \\
\text { contractors }\end{array}$ & $D>0$ \\
\hline$K$ & $\begin{array}{l}\text { Market demand for recycled products of downstream } \\
\text { construction contractors (considering the upstream and } \\
\text { downstream industrial chain between the construction } \\
\text { contractors and recycled products dealers) }\end{array}$ & $D \geq K \geq 0$ \\
\hline$\lambda$ & $\begin{array}{l}\text { Proportion of the demand for recycled products of construction } \\
\text { contractors in the total demand for building materials, including } \\
\text { recycled products and traditional building materials }\end{array}$ & $1 \geq \lambda \geq 0$ \\
\hline
\end{tabular}

The assumptions and definitions of the decision variables are as follows:

$x_{1}$ : Output of construction wastes disposed to recycling by construction contractors;

$x_{2}$ : Output of recycled products of a construction waste recycling enterprise;

$x_{3}$ : Volume of sales of recycled products by the recycled products dealers.

Note: Initial variables of $x_{1}, x_{2}$ and $x_{3}$ are $x_{10}, x_{20}$ and $x_{30}$. 


\section{Construction of the co-evolution model based on Lotka-Volterra}

According to Zhou, the co-evolution between upstream and downstream enterprises in the residential industry chain is studied, and an ecological Lotka-Volterra model is used as a reference to build a co-evolution model between upstream (design units, product suppliers) and downstream enterprises (developers and properties) [32]. Based on the studies of Green [27], Gao and Xie [13], the industrial chain of construction waste recycling, composed of construction contractors, construction waste recycling enterprises, and recycled products dealers, is considered as the research object, and a co-evolution model is constructed under the condition of abundant supply of building materials (including recycled products and traditional building materials), as shown in (1) [13] [27]:

$$
\left\{\begin{array}{l}
\frac{d x_{1}}{d t}=\beta_{1} x_{1}\left(D-x_{1}\right)\left(\varepsilon_{1} x_{2}-x_{1}\right) \\
\frac{d x_{2}}{d t}=\beta_{2} x_{2}\left(\tau_{1} x_{1}-x_{2}\right)\left(\varepsilon_{2} x_{3}-x_{2}\right) \\
\frac{d x_{3}}{d t}=\beta_{3} z\left(\tau_{2} x_{2}-x_{3}\right)\left(K-x_{3}\right) \\
K=\lambda D
\end{array}\right.
$$

Where,

$\frac{d x_{1}}{d t}=$ rate of change in the output of construction waste recycled by a construction contractor (reflecting the growth rate of the construction contractor);

$$
\frac{d x_{2}}{d t}=\text { rate of change in the output of recycled }
$$
products of a construction waste recycling enterprise (reflecting the growth rate of the construction waste recycling enterprise);

$$
\frac{d x_{3}}{d t}=\text { rate of change in sales of recycled products by }
$$
a recycled products dealer (reflecting the growth rate of the recycled products dealer);

$\left(\varepsilon_{1} x_{2}-x_{1}\right)=$ increased demand for construction waste of the downstream construction waste recycling enterprises (considering the upstream and downstream industrial chain between construction contractors and construction waste recycling enterprises); The condition $\left(\varepsilon_{1} x_{2}-x_{1}\right) \geq 0$ should always be met; Otherwise, the output of the construction waste from construction contractors will tend to 0 ;

$$
\left(\tau_{1} x_{1}-x_{2}\right)=\text { increased supply of construction waste }
$$

from upstream construction contractors (considering the upstream and downstream industrial chain between construction contractors and construction waste recycling enterprises); The condition $\left(\tau_{1} x_{1}-x_{2}\right) \geq 0$ should always be met; Otherwise, the output of recycled products of the construction waste recycling enterprises will tend to 0 ;

$$
\left(\varepsilon_{2} x_{3}-x_{2}\right)=\text { increased market demand for recycled }
$$
products of downstream recycled products dealers (considering the upstream and downstream industrial chain between the construction waste recycling enterprises and recycled product dealers); The condition $\left(\varepsilon_{2} x_{3}-x\right)_{2} \geq 0$ should always be met; Otherwise, the output of recycled products of the construction waste recycling enterprises will tend to 0 ;

$$
\left(\tau_{2} x_{2}-x_{3}\right)=\text { increased supply of recycled products }
$$
of the upstream construction waste recycling enterprises (considering the upstream and downstream industrial chain between construction waste recycling enterprises and recycled products dealers); The condition $\left(\tau_{2} x_{2}-x_{3}\right) \geq 0$ should always be met; Otherwise, the sales volume of recycled products of the recycled products dealers will tend to 0 .

Let $\frac{d x_{1}}{d t}, \frac{d x_{2}}{d t}, \frac{d x_{3}}{d t}$ be 0 . Then, the steady-state solutions of $\left(x_{1}, x_{2}, x_{3}\right)$ in the model are $A_{1}(0,0,0)$,
$A_{2}(0,0, \lambda D)$
$A_{3}(D, 0,0)$
$A_{4}(D, 0, \lambda D)$
$A_{5}\left(D, \tau_{1} D, 0\right) \quad, \quad A_{6}\left(D, \frac{D}{\varepsilon_{1}}, 0\right)$
$A_{7}\left(0, \varepsilon_{2} \lambda D, \lambda D\right)$ 
$A_{8}\left(0, \frac{\lambda D}{\tau_{2}}, \lambda D\right), \quad A_{9}\left(D, \tau_{1} D, \lambda D\right), \quad A_{10}\left(D, \varepsilon_{2} \lambda D, \lambda D\right)$,

$A_{11}\left(\varepsilon_{1} \varepsilon_{2} \lambda D, \varepsilon_{2} \lambda D, \lambda D\right)$, and $A_{12}\left(D, \tau_{1} D, \tau_{1} \tau_{2} D\right)$.

Among them, the isoclinic of the model is shown in

\section{Judgment of stability of steady-state solutions}

According to the Jacobian matrix proposed by Friedman (1991), the stability judgment is made on the local equilibrium

points $A_{1}, A_{2}, A_{3}, A_{4}, A_{5}, A_{6}, A_{7}, A_{8}, A_{9}, A_{10}$ of the above 10 steadystate solutions of the co-evolution model for the industrial chain of construction waste recycling, and the partial derivatives of the $\frac{d x_{1}}{d t}, \frac{d x_{2}}{d t}, \frac{d x_{3}}{d t}$ pairs $\left(x_{1}, x_{2}, x_{3}\right)$ in Equation (1) are obtained by partial differentiation, respectively, from which the corresponding Jacobian matrix is obtained ((3) - (11)):

$\frac{\partial\left(\frac{d x_{1}}{d t}\right)}{\partial x_{1}}=\beta_{1}\left(D-x_{1}\right)\left(\varepsilon_{1} x_{2}-x_{1}\right)-\beta_{1} y\left(\varepsilon_{1} x_{2}-x_{1}\right)-\beta_{1} x_{1}\left(D-x_{1}\right)$

$\frac{\partial\left(\frac{d x_{1}}{d t}\right)}{\partial x_{2}}=\beta_{1} x_{1}\left(D-x_{1}\right) \varepsilon_{1}$

$$
\frac{\partial\left(\frac{d x_{1}}{d t}\right)}{\partial x_{3}}=0
$$

$$
\frac{\partial\left(\frac{d x_{2}}{d t}\right)}{\partial x_{1}}=\beta_{2} x_{2} \tau_{1}\left(\varepsilon_{2} x_{3}-x_{2}\right)
$$

$\frac{\partial\left(\frac{d x_{2}}{d t}\right)}{\partial x_{2}}=\beta_{2}\left(\tau_{1} x_{1}-x_{2}\right)\left(\varepsilon_{2} x_{3}-x_{2}\right)-\beta_{2} x\left(\varepsilon_{2} x_{3}-x_{2}\right)-\beta_{2} x_{2}$
$\frac{\partial\left(\frac{d x_{2}}{d t}\right)}{\partial x_{3}}=\beta_{2} x_{2}\left(\tau_{1} x_{1}-x_{2}\right) \varepsilon_{2}$

(8)
(2):

$$
\left\{\begin{array}{l}
\beta_{1} x_{1}\left(D-x_{1}\right)\left(\varepsilon_{1} x_{2}-x_{1}\right)=0 \\
\beta_{2} x_{2}\left(\tau_{1} x_{1}-x_{2}\right)\left(\varepsilon_{2} x_{3}-x_{2}\right)=0 \\
\beta_{3} x_{3}\left(\tau_{2} x_{2}-x_{3}\right)\left(K-x_{3}\right)=0
\end{array}\right.
$$

$$
\frac{\partial\left(\frac{d x_{3}}{d t}\right)}{\partial x_{1}}=0
$$

$$
\frac{\partial\left(\frac{d x_{3}}{d t}\right)}{\partial x_{2}}=\beta_{3} x_{3} \tau_{2}\left(K-x_{3}\right)
$$

$$
\frac{\partial\left(\frac{d x_{3}}{d t}\right)}{\partial x_{3}}=\beta_{3}\left(\tau_{2} x_{2}-x_{3}\right)\left(K-x_{3}\right)-\beta_{3} x_{3}\left(K-x_{3}\right)-\beta_{3} x_{3}\left(\tau_{2} x_{2}-x_{3}\right)
$$

To summarize, the Jacobian matrix is

$$
J=\left(\begin{array}{lll}
m_{11} & m_{12} & m_{13} \\
m_{21} & m_{22} & m_{23} \\
m_{31} & m_{32} & m_{33}
\end{array}\right) .
$$

After

organizing:

$$
J=\left(\begin{array}{lll}
\frac{\partial\left(\frac{d x_{1}}{d t}\right)}{\partial x_{1}} & \frac{\partial\left(\frac{d x_{1}}{d t}\right)}{\partial x_{2}} & \frac{\partial\left(\frac{d x_{1}}{d t}\right)}{\partial x_{3}} \\
\frac{\partial\left(\frac{d x_{2}}{d t}\right)}{\partial x_{1}} & \frac{\partial\left(\frac{d x_{2}}{d t}\right)}{\partial x_{2}} & \frac{\partial\left(\frac{d x_{2}}{d t}\right)}{\partial x_{3}} \\
\frac{\partial\left(\frac{d x_{3}}{d t}\right)}{\partial x_{1}} & \frac{\partial\left(\frac{d x_{3}}{d t}\right)}{\partial x_{2}} & \frac{\partial\left(\frac{d x_{3}}{d t}\right)}{\partial x_{3}}
\end{array}\right) ;
$$

Thus, the Jacobian matrix can be used to judge matrix indicates that when $\operatorname{det}(J)>0$ and $\operatorname{tr}(J)<0$, the steady-state solutions will approach the stable state. Therefore, the stability of the local equilibrium points $A_{1}, A_{2}, A_{3}, A_{4}, A_{5}, A_{6}, A_{7}, A_{8}, A_{9}, A_{10} \quad$ of 10 steady-state solutions of the co-evolution model for the construction waste recycling industrial chain is determined as shown in Table II. 


\begin{tabular}{|c|c|c|c|}
\hline $\begin{array}{l}\text { Local equilibrium } \\
\text { points }\end{array}$ & $\operatorname{det}(J)$ & $\operatorname{tr}(J)$ & $\begin{array}{l}\text { State of } \\
\text { stability }\end{array}$ \\
\hline$A_{1}(0,0,0)$ & 0 & 0 & Saddle point \\
\hline$A_{2}(0,0, \lambda D)$ & 0 & $\beta_{3} \lambda^{2} D^{2}$ & $\begin{array}{l}\text { Unstable } \\
\text { point }\end{array}$ \\
\hline$A_{3}(D, 0,0)$ & 0 & $\beta_{1} D^{2}>0$ & $\begin{array}{l}\text { Unstable } \\
\text { point }\end{array}$ \\
\hline$A_{4}(D, 0, \lambda D)$ & $\beta_{1} \beta_{2} \beta_{3} \tau_{1} \varepsilon_{2} \lambda^{3} D^{6}$ & $\begin{array}{l}\beta_{2} \tau_{1} \varepsilon_{2} \lambda D^{2}+\beta_{1} D^{2} \\
+\beta_{3} \lambda^{2} D^{2}\end{array}$ & $\begin{array}{l}\text { Unstable } \\
\text { point }\end{array}$ \\
\hline$A_{5}\left(D, \tau_{1} D, 0\right)$ & $\begin{array}{l}\beta_{1} \beta_{2} \beta_{3} \tau_{1}^{3} \tau_{2} \lambda D^{6} \\
\left(1-\varepsilon_{1} \tau_{1}\right)\end{array}$ & $\begin{array}{l}\beta_{2} \tau_{1}^{2} D^{2}-\beta_{1} D\left(\varepsilon_{1} \tau_{1} D-D\right) \\
+\beta_{3} \tau_{1} \tau_{2} \lambda D^{2}\end{array}$ & $\begin{array}{l}\text { Unstable } \\
\text { point }\end{array}$ \\
\hline$A_{6}\left(D, \frac{D}{\varepsilon_{1}}, 0\right)$ & 0 & $\beta_{2} \frac{D^{2}}{\varepsilon_{1}^{2}}+2 \beta_{2} \frac{D^{2}}{\varepsilon_{1}}\left(\frac{1}{\varepsilon_{1}}-\tau_{1}\right)$ & $\begin{array}{l}\text { Unstable } \\
\text { point }\end{array}$ \\
\hline$A_{7}\left(0, \varepsilon_{2} \lambda D, \lambda D\right)$ & $\begin{array}{l}\beta_{1} \beta_{2} \beta_{3} \varepsilon_{1} \varepsilon_{2}^{3} \lambda^{5} D^{6} \\
\left(1-\tau_{2} \varepsilon_{2}\right)\end{array}$ & $\begin{array}{l}\beta_{2} \varepsilon_{2}^{2} \lambda^{2} D^{2}+\beta_{1} \varepsilon_{1} \varepsilon_{2} \lambda D^{2} \\
+\beta_{3} \lambda^{2} D^{2}\left(1-\varepsilon_{2} \tau_{2}\right)\end{array}$ & $\begin{array}{l}\text { Unstable } \\
\text { point }\end{array}$ \\
\hline$A_{8}\left(0, \frac{\lambda D}{\tau_{2}}, \lambda D\right)$ & 0 & $\begin{array}{l}2 \beta_{2} \frac{\lambda^{2} D^{2}}{\tau_{2}}\left(\frac{1}{\tau_{2}}-\varepsilon_{2}\right)+\beta_{2} \\
\frac{\lambda^{2} D^{2}}{\tau_{2}^{2}}+\beta_{1} \varepsilon_{1} D^{2} \frac{\lambda}{\tau_{2}}\end{array}$ & $\begin{array}{l}\text { Unstable } \\
\text { point }\end{array}$ \\
\hline$A_{9}\left(D, \tau_{1} D, \lambda D\right)$ & $\begin{array}{l}\beta_{1} \beta_{2} \beta_{3} \lambda \tau_{1} D^{6} \\
\left(\varepsilon_{2} \lambda-\tau_{1}\right)\left(1-\varepsilon_{1} \tau_{1}\right) \\
\left(\tau_{1} \tau_{2}-\lambda\right)\end{array}$ & $\begin{array}{l}-\beta_{2} \tau_{1} D^{2}\left(\varepsilon_{2} \lambda-\tau_{1}\right)-\beta_{1} D^{2} \\
\left(\varepsilon_{1} \tau_{1}-1\right)-\beta_{3} \lambda D^{2}\left(\tau_{1} \tau_{2}-\lambda\right)\end{array}$ & - \\
\hline$A_{10}\left(D, \varepsilon_{2} \lambda D, \lambda D\right)$ & $\begin{array}{l}\beta_{1} \beta_{2} \beta_{3} \varepsilon_{2} \lambda^{3} D^{6} \\
\left(\tau_{1}-\varepsilon_{2} \lambda\right)\left(1-\varepsilon_{2} \tau_{2}\right) \\
\left(\varepsilon_{1} \varepsilon_{2} \lambda-1\right)\end{array}$ & $\begin{array}{l}-\beta_{2} \varepsilon_{2} \lambda D^{2}\left(\tau_{1}-\varepsilon_{2} \lambda\right)- \\
D^{2}\left(\varepsilon_{1} \varepsilon_{2} \lambda-1\right)-\lambda^{2} D^{2} \\
\left(\varepsilon_{2} \tau_{2}-1\right)\end{array}$ & - \\
\hline$A_{11}\left(\begin{array}{l}\varepsilon_{1} \varepsilon_{2} \lambda D, \varepsilon_{2} \lambda D, \\
\lambda D\end{array}\right)$ & $\begin{array}{l}-\beta_{1} \beta_{2} \beta_{3} \varepsilon_{1} \varepsilon_{2}{ }^{3} \lambda^{5} D^{6} \\
\left(\tau_{1} \varepsilon_{1}-1\right)\left(1-\varepsilon_{1} \varepsilon_{2} \lambda\right) \\
\left(\tau_{2} \varepsilon_{2}-1\right)\end{array}$ & $\begin{array}{l}-\beta_{2} \varepsilon_{2}^{2} \lambda^{2} D^{2}\left(\tau_{1} \varepsilon_{1}-1\right) \\
-\beta_{1} \varepsilon_{1} \varepsilon_{2} \lambda D^{2}\left(1-\varepsilon_{1} \varepsilon_{2} \lambda\right) \\
-\beta_{3} \lambda^{2} D^{2}\left(\tau_{2} \varepsilon_{2}-1\right)\end{array}$ & - \\
\hline$A_{12}\left(D, \tau_{1} D, \tau_{1} \tau_{2} D\right)$ & $\begin{array}{l}-\beta_{1} \beta_{2} \beta_{3} \tau_{1}^{3} \tau_{2} D^{6} \\
\left(\varepsilon_{2} \tau_{2}-1\right)\left(\varepsilon_{1} \tau_{1}-1\right) \\
\left(\lambda-\tau_{1} \tau_{2}\right)\end{array}$ & $\begin{array}{l}-\beta_{2} \tau_{1}^{2} D^{2}\left(\tau_{2} \varepsilon_{2}-1\right) \\
-\beta_{1} D^{2}\left(\varepsilon_{1} \tau_{1}-1\right) \\
-\beta_{3} \tau_{1} \tau_{2} D^{2}\left(\lambda-\tau_{1} \tau_{2}\right)\end{array}$ & - \\
\hline
\end{tabular}

(Note: $\operatorname{det}(J)=m_{11} m_{22} m_{33}-m_{12} m_{23} m_{31}-m_{13} m_{21} m_{32}, \operatorname{tr}(J)=m_{11}+m_{22}+m_{33}, \quad K=\lambda D$, “-” indicates uncertain stability). 
Table II shows that the stability of $A_{9}, A_{10}, A_{11}, A_{12}$ cannot be judged. Thus, the stability judgment of $A_{9}\left(D, \tau_{1} D, \lambda D\right) \quad, \quad A_{10}\left(D, \varepsilon_{2} \lambda D, \lambda D\right)$ $A_{11}\left(\varepsilon_{1} \varepsilon_{2} \lambda D, \varepsilon_{2} \lambda D, \lambda D\right)$, and $A_{12}\left(D, \tau_{1} D, \tau_{1} \tau_{2} D\right)$ can be analyzed as follows:

(1) Stability judgment and analysis of $A_{9}\left(D, \tau_{1} D, \lambda D\right)$

Case 1:

When

$\left\{\begin{array}{l}\beta_{1} \beta_{2} \beta_{3}\left(\varepsilon_{2} \lambda-\tau_{1}\right)\left(1-\varepsilon_{1} \tau_{1}\right)\left(\tau_{1} \tau_{2}-\lambda\right)>0 \\ \beta_{2} \tau_{1}\left(\varepsilon_{2} \lambda-\tau_{1}\right)+\beta_{1}\left(\varepsilon_{1} \tau_{1}-1\right)+\beta_{3} \lambda\left(\tau_{1} \tau_{2}-\lambda\right)>0\end{array}, \quad\right.$ the local equilibrium point $A_{9}$ is a stable point.

Case 2:

When $\left\{\begin{array}{l}\beta_{1} \beta_{2} \beta_{3}\left(\varepsilon_{2} \lambda-\tau_{1}\right)\left(1-\varepsilon_{1} \tau_{1}\right)\left(\tau_{1} \tau_{2}-\lambda\right)=0 \\ \beta_{2} \tau_{1}\left(\varepsilon_{2} \lambda-\tau_{1}\right)+\beta_{1}\left(\varepsilon_{1} \tau_{1}-1\right)+\beta_{3} \lambda\left(\tau_{1} \tau_{2}-\lambda\right)=0\end{array}, \quad\right.$ the local equilibrium point $A_{9}$ is a saddle point.

Case 3: If neither Case 1 nor Case 2 is met, the local equilibrium point $A_{9}$ is an unstable point.

(2) Stability judgment and analysis of $A_{10}\left(D, \varepsilon_{2} \lambda D, \lambda D\right)$

\section{Case} 4:

When

$\left\{\begin{array}{l}\beta_{1} \beta_{2} \beta_{3}\left(\varepsilon_{2} \lambda-\tau_{1}\right)\left(1-\varepsilon_{2} \tau_{2}\right)\left(1-\varepsilon_{1} \varepsilon_{2} \lambda\right)>0 \\ \beta_{2} \varepsilon_{2} \lambda\left(\varepsilon_{2} \lambda-\tau_{1}\right)+\beta_{1}\left(1-\varepsilon_{1} \varepsilon_{2} \lambda\right)+\beta_{3} \lambda^{2}\left(1-\varepsilon_{2} \tau_{2}\right)<0\end{array}\right.$, the

local equilibrium point $A_{10}$ is a stable point.

Case 5:

When

$\left\{\begin{array}{l}\beta_{1} \beta_{2} \beta_{3}\left(\varepsilon_{2} \lambda-\tau_{1}\right)\left(1-\varepsilon_{2} \tau_{2}\right)\left(1-\varepsilon_{1} \varepsilon_{2} \lambda\right)=0 \\ \beta_{2} \varepsilon_{2} \lambda\left(\varepsilon_{2} \lambda-\tau_{1}\right)+\beta_{1}\left(1-\varepsilon_{1} \varepsilon_{2} \lambda\right)+\beta_{3} \lambda^{2}\left(1-\varepsilon_{2} \tau_{2}\right)=0\end{array}\right.$, the

local equilibrium point $A_{10}$ is a saddle point.

Case 6: If neither Case 4 nor Case 5 is met, the local equilibrium point $A_{10}$ is an unstable point.

(3) Stability judgment and analysis of $A_{11}\left(\varepsilon_{1} \varepsilon_{2} \lambda D, \varepsilon_{2} \lambda D, \lambda D\right)$

Case 7:

When

$\left\{\begin{array}{l}\beta_{1} \beta_{2} \beta_{3} \varepsilon_{1} \varepsilon_{2}{ }^{3} \lambda^{5}\left(\tau_{1} \varepsilon_{1}-1\right)\left(1-\varepsilon_{1} \varepsilon_{2} \lambda\right)\left(\tau_{2} \varepsilon_{2}-1\right)<0 \\ -\beta_{2} \varepsilon_{2}{ }^{2} \lambda^{2}\left(\tau_{1} \varepsilon_{1}-1\right)-\beta_{1} \varepsilon_{1} \varepsilon_{2} \lambda\left(1-\varepsilon_{1} \varepsilon_{2} \lambda\right)-\beta_{3} \lambda^{2}\left(\tau_{2} \varepsilon_{2}-1\right)<0\end{array}\right.$
, the local equilibrium point $A_{11}$ is a stable point.

Case

8:

When

$\left\{\begin{array}{l}\beta_{1} \beta_{2} \beta_{3} \varepsilon_{1} \varepsilon_{2}{ }^{3} \lambda^{5}\left(\tau_{1} \varepsilon_{1}-1\right)\left(1-\varepsilon_{1} \varepsilon_{2} \lambda\right)\left(\tau_{2} \varepsilon_{2}-1\right)=0 \\ -\beta_{2} \varepsilon_{2}{ }^{2} \lambda^{2}\left(\tau_{1} \varepsilon_{1}-1\right)-\beta_{1} \varepsilon_{1} \varepsilon_{2} \lambda\left(1-\varepsilon_{1} \varepsilon_{2} \lambda\right)-\beta_{3} \lambda^{2}\left(\tau_{2} \varepsilon_{2}-1\right)=0\end{array}\right.$

, the local equilibrium point $A_{11}$ is a saddle point.

Case 9: If neither Case 7 nor Case 8 is met, the local equilibrium point $A_{11}$ is an unstable point.

(4) Stability judgment and analysis of $A_{12}\left(D, \tau_{1} D, \tau_{1} \tau_{2} D\right)$

Case

10:

When

$\left\{\begin{array}{l}-\beta_{1} \beta_{2} \beta_{3} \tau_{1}^{3} \tau_{2}\left(\tau_{2} \varepsilon_{2}-1\right)\left(\varepsilon_{1} \tau_{1}-1\right)\left(\lambda-\tau_{1} \tau_{2}\right)>0 \\ -\beta_{2} \tau_{1}^{2}\left(\tau_{2} \varepsilon_{2}-1\right)-\beta_{1}\left(\varepsilon_{1} \tau_{1}-1\right)-\beta_{3} \tau_{1} \tau_{2}\left(\lambda-\tau_{1} \tau_{2}\right)<0\end{array}\right.$, the local equilibrium point $A_{12}$ is a stable point.

Case

11:

When

$\left\{\begin{array}{l}-\beta_{1} \beta_{2} \beta_{3} \tau_{1}^{3} \tau_{2}\left(\tau_{2} \varepsilon_{2}-1\right)\left(\varepsilon_{1} \tau_{1}-1\right)\left(\lambda-\tau_{1} \tau_{2}\right)=0 \\ -\beta_{2} \tau_{1}^{2}\left(\tau_{2} \varepsilon_{2}-1\right)-\beta_{1}\left(\varepsilon_{1} \tau_{1}-1\right)-\beta_{3} \tau_{1} \tau_{2}\left(\lambda-\tau_{1} \tau_{2}\right)=0\end{array}\right.$, the

local equilibrium point $A_{12}$ is a saddle point.

Case 12: If neither Case 10 nor Case 11 is met, the local equilibrium point $A_{12}$ is an unstable point.

\section{F. Analysis of the synergy effect}

Discussions on Cases 1, 4, 7, and 10:

a) When $\tau_{1}>\varepsilon_{2} \lambda, \quad x=\varepsilon_{2} \lambda D, z=\lambda D$.

Under conditions where the parameter meets Case 1 or 4 or 7 or 10 , if $\varepsilon_{1} \varepsilon_{2} \lambda<1$, then $y=\varepsilon_{1} \varepsilon_{2} \lambda D$, that is, $A_{11}\left(\varepsilon_{1} \varepsilon_{2} \lambda D, \varepsilon_{2} \lambda D, \lambda D\right)$ is a stable local equilibrium point; If $\varepsilon_{1} \varepsilon_{2} \lambda>1$, then $y=D$, that is, $A_{10}\left(D, \varepsilon_{2} \lambda D, \lambda D\right)$ is a stable local equilibrium point; If $\varepsilon_{1} \varepsilon_{2} \lambda=1 \quad, \quad$ then $\quad A_{11}\left(\varepsilon_{1} \varepsilon_{2} \lambda D, \varepsilon_{2} \lambda D, \lambda D\right) \quad$ or $A_{10}\left(D, \varepsilon_{2} \lambda D, \lambda D\right)$ is a stable local equilibrium point.

b) When $\tau_{1}=\varepsilon_{2} \lambda$, under conditions where the parameter meets Case 1 or 4 or 7 or 10 , if $\varepsilon_{1} \varepsilon_{2} \lambda>1$ and $\tau_{1} \tau_{2}>\lambda$, then $A_{9}\left(D, \tau_{1} D, \lambda D\right)$ or $A_{10}\left(D, \varepsilon_{2} \lambda D, \lambda D\right)$ is a stable local equilibrium point; If $\varepsilon_{1} \varepsilon_{2} \lambda<1$ and 
$\tau_{1} \tau_{2}>\lambda$, then $A_{11}\left(\varepsilon_{1} \varepsilon_{2} \lambda D, \varepsilon_{2} \lambda D, \lambda D\right)$ is a stable local equilibrium point; If $\varepsilon_{1} \varepsilon_{2} \lambda>1$ and $\tau_{1} \tau_{2}<\lambda$, then $A_{12}\left(D, \tau_{1} D, \tau_{1} \tau_{2} D\right)$ is a stable local equilibrium point; If $\varepsilon_{1} \varepsilon_{2} \lambda=1$ and $\tau_{1} \tau_{2}=\lambda$, then $A_{9}\left(D, \tau_{1} D, \lambda D\right)$, or $A_{10}\left(D, \varepsilon_{2} \lambda D, \lambda D\right)$, or $A_{11}\left(\varepsilon_{1} \varepsilon_{2} \lambda D, \varepsilon_{2} \lambda D, \lambda D\right)$, or $A_{12}\left(D, \tau_{1} D, \tau_{1} \tau_{2} D\right)$ is a stable local equilibrium point.

\section{NUMERICAL SIMULATIONS}

Based on the above analysis of (a), (b), and (c), MATLAB (R2015a) was used to simulate the co-evolution of the construction waste recycling industrial chain in Cases 1, 4, 7, and 10, aiming to verify the correctness of the above local equilibrium point stability analysis and study the influence of various factors on the co-evolution process of the industrial chain, including simulation analysis of tendencies and sensitivity.

\section{A. Co-evolution tendency and sensitivity analyses under}

\section{Case 1 conditions}

(1) Analysis of the tendency of co-evolution of construction waste recycling industrial chain

The industrial chain of construction waste recycling is considered as the research object. The initial output of enterprises (including construction waste recycling enterprises, recycled products dealers, and construction contractors), market demand $K$ of construction contractors on recycled products, and maximum production $D$ of construction waste (including waste from recycled products and traditional building materials as well as waste such as residues) of construction contractors are assigned in the co-evolution model of c) When $\tau_{1}<\varepsilon_{2} \lambda$, then $x=\tau_{1} D, y=D$.

Under conditions where the parameter meets Case 1 or 4 or 7 or 10 , if $\tau_{1} \tau_{2}<\lambda$, then $z=\tau_{1} \tau_{2} D$, that is, $A_{12}\left(D, \tau_{1} D, \tau_{1} \tau_{2} D\right)$ is a stable local equilibrium point; If $\tau_{1} \tau_{2}>\lambda$, then $z=\lambda D$, that is, $A_{9}\left(D, \tau_{1} D, \lambda D\right)$ is a stable local equilibrium point; If $\tau_{1} \tau_{2}=\lambda$, then $A_{9}\left(D, \tau_{1} D, \lambda D\right)$ or $A_{12}\left(D, \tau_{1} D, \tau_{1} \tau_{2} D\right)$ is a stable local equilibrium point.

$$
\begin{aligned}
& x_{10}=164.6, \quad x_{20}=123.45, \quad x_{30}=87.5, \lambda=0.64 \text { (i.e., ) } \\
& K=160 \quad D=250, \quad \beta_{1}=0.5, \quad \beta_{2}=0.15, \quad \beta_{3}=0.2, \\
& \varepsilon_{1}=1.7, \quad \varepsilon_{2}=0.8, \quad \tau_{1}=0.6 \text {, and } \tau_{2}=1.4 .
\end{aligned}
$$

Based on the above data, the stability analysis of the local equalization points $A_{9}\left(\tau_{1} D, D, \lambda D\right)$, $A_{10}\left(\varepsilon_{2} \lambda D, D, \lambda D\right) \quad, \quad A_{11}\left(\varepsilon_{2} \lambda D, \varepsilon_{1} \varepsilon_{2} \lambda D, \lambda D\right) \quad, \quad$ and $A_{12}\left(\tau_{1} D, D, \tau_{1} \tau_{2} D\right)$ can be performed. The results show that if the conditions in Case 1 are met $\left\{\begin{array}{l}\beta_{1} \beta_{2} \beta_{3}\left(\varepsilon_{2} \lambda-\tau_{1}\right)\left(1-\varepsilon_{1} \tau_{1}\right)\left(\tau_{1} \tau_{2}-\lambda\right)>0 \\ \beta_{2} \tau_{1}\left(\varepsilon_{2} \lambda-\tau_{1}\right)+\beta_{1}\left(\varepsilon_{1} \tau_{1}-1\right)+\beta_{3} \lambda\left(\tau_{1} \tau_{2}-\lambda\right)>0\end{array}\right.$, then $\tau_{1}>\varepsilon_{2} \lambda$ and $\varepsilon_{1} \varepsilon_{2} \lambda<1$. Therefore, $A_{11}(128,217.6,160)$ is a stability point in the co-evolution process of the construction waste recycling industrial chain.

The simulation validation was performed using 0.005 as the simulation cycle. The simulation results of the coevolution of the industrial chain of construction waste recycling meeting the conditions of Case 1 are shown in Fig. 2. 


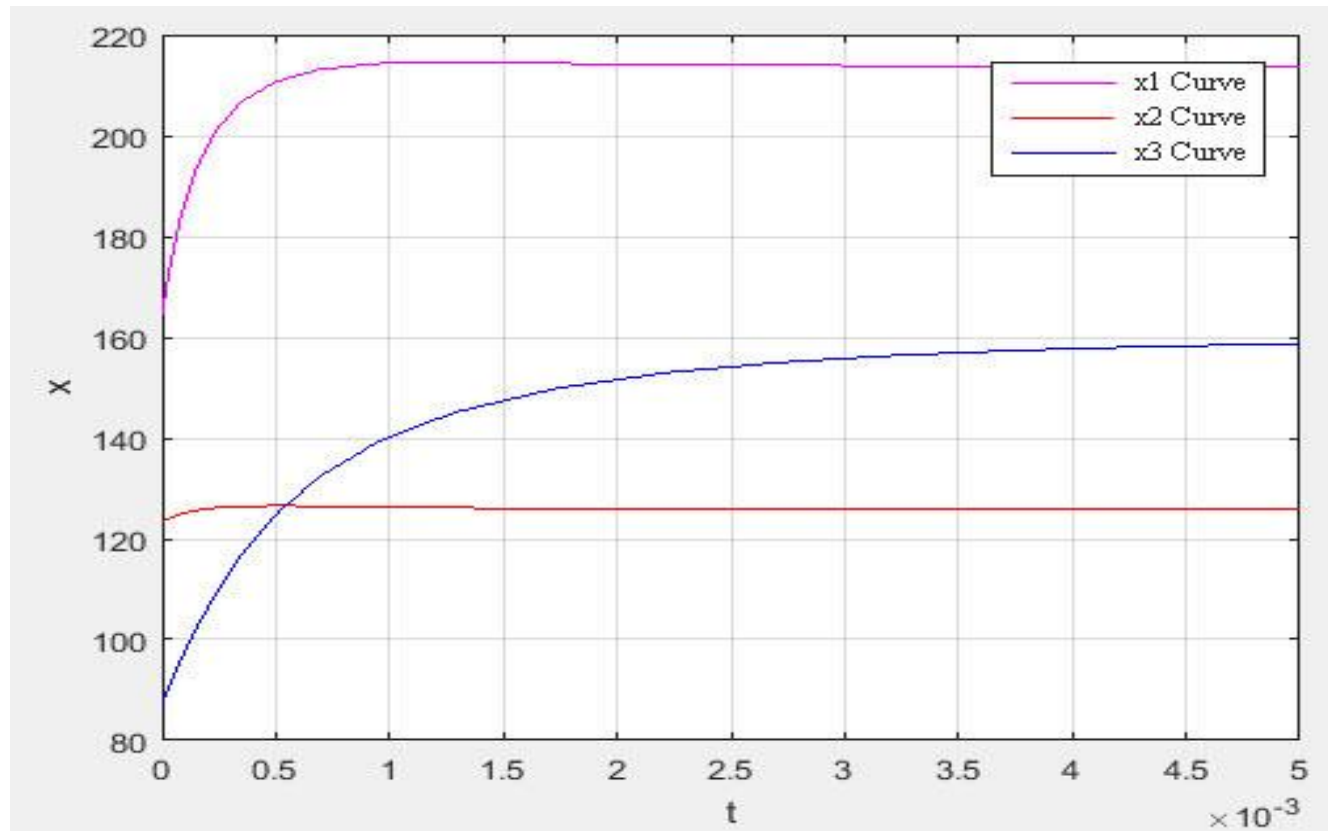

Fig. 2 Simulation of the co-evolution of the industrial chain of construction waste recycling meeting the conditions of Case 1 (Note: $\mathrm{x} 1$ curve represents y, i.e., the output of construction waste from construction contractors; $x 2$ curve represents $x$, i.e., the output of recycled products of construction waste recycling enterprises; $x 3$ curve represents z, i.e. the sales volume of the recycled products by the recycled products dealers. The same applies to Figs. 5 and 8 ).

Analysis of Fig. 2 shows that the output of recycled products of construction waste recycling enterprises gradually increases from the initial output ranging from 123.45 to 124.80 with time, reaching a stable state at $0.3 \times 10^{-3}$, and the growth rate of the output gradually decreases and tends to be stable at 0 ; The output of construction wastes of construction contractors increases gradually from an initial output ranging from 164.6 to 213.9 with time, reaching a stable state at $1 \times 10^{-3}$, and the growth rate of construction waste output gradually decreases and becomes stable at 0 ; The sales volume of recycled products of the recycled products dealers gradually increases from an initial output of 87.5 to 158.7 over time, reaching a stable state at $5 \times 10^{-3}$, and the growth rate of construction waste output gradually decreases and becomes stable at 0 . Thus, it is clear that the simulation results are in accordance with the analysis results.

(2) Sensitivity analysis of co-evolution of the construction waste recycling industrial chain

Assuming that other conditions remain unchanged, that is $x_{10}=164.6, x_{20}=123.45, x_{30}=87.5, K=160$, $D=250, \varepsilon_{1}=1.7, \varepsilon_{2}=0.8, \tau_{1}=0.6$, and $\tau_{2}=1.4$, and increasing and reducing by $30 \%$ the natural growth rates $\beta_{1}=0.5, \beta_{2}=0.15$, and $\beta_{3}=0.2$, the simulation results of the co-evolution sensitivity of the natural growth rate of the construction waste recycling industrial chain are shown in Fig. 3. 


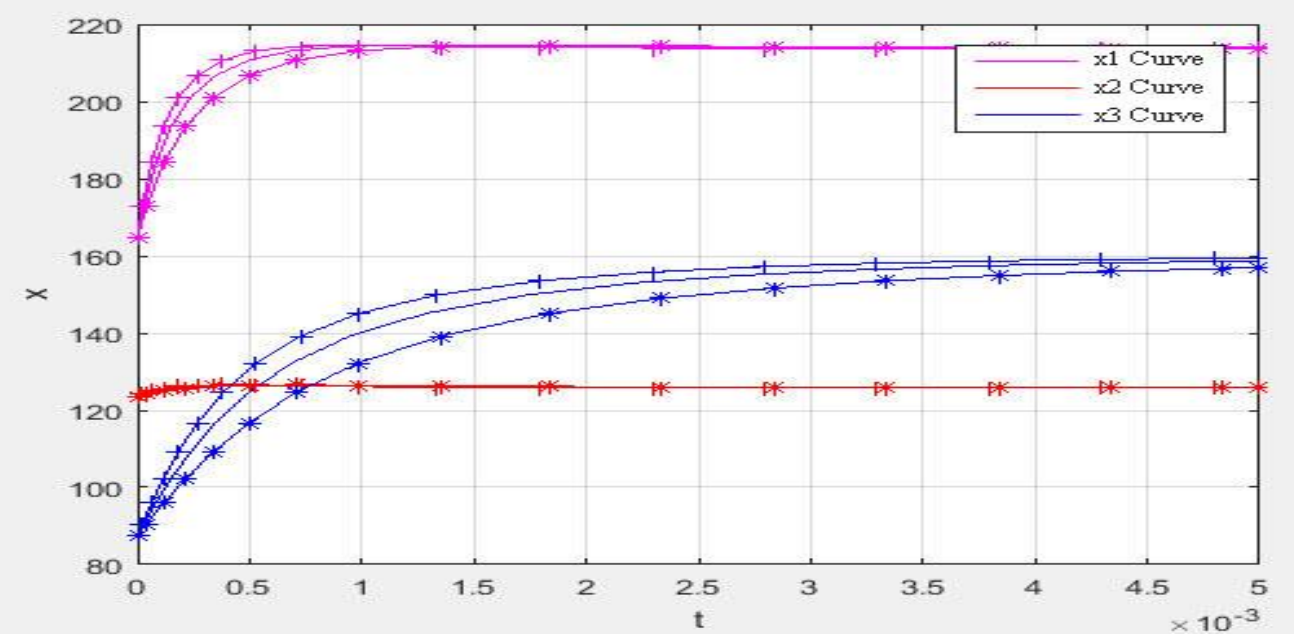

Fig. 3 Sensitivity analysis of the natural growth rate of the construction waste recycling industrial chain meeting the conditions of Case 1 (Note: "+solid line" means an increase of 30\%; "* solid line" means a reduction of 30\%; "Solid line" indicates the original value; Pink indicates y, i.e., the output of construction contractors; Red indicates x, i.e., the output of construction waste recycling enterprises; And blue indicates z, i.e., the output of recycled products dealers. The same applies to Figs 4, 6, 7, 9, and 10)

Analysis of Fig. 3 shows that when the natural growth rates are increased by $30 \%$, the stable values of the output of recycled products from the construction waste recycling enterprises, the output of construction waste from the construction contractors, and the sales of recycled products by the recycled products dealers are 128, 217.6 and 160, respectively, which are the same as the original stable values; The evolution rates of these three parameters are accelerated at the same time, and the evolution rate is evident for the construction wastes from construction contractors and sales of recycled products by the recycled products dealers, but not for the recycled product output of the construction waste recycling enterprises. When the natural growth rates are reduced by $30 \%$, the stable values of the output of recycled products from the construction waste recycling enterprises, the output of construction waste from the construction contractors and the sales of recycled products by the recycled products dealers are 128 ,
217.6, and 160, respectively, which are the same as the original stable values. The evolution rates of the three parameters are decelerated at the same time, and the evolution rate is evident for the construction wastes from construction contractors and sales of recycled products by the recycled products dealers, but not for the recycled product output of the construction waste recycling enterprises.

Assuming that other conditions remain unchanged, that is $x_{10}=164.6, x_{20}=123.45, x_{30}=87.5, K=160$, $D=250, \beta_{1}=0.5, \quad \beta_{2}=0.15$, and $\beta_{3}=0.2$, and increasing and reducing by $30 \%$ the conversion coefficients $\varepsilon_{1}=1.7, \varepsilon_{2}=0.8, \tau_{1}=0.6$ and $\tau_{2}=1.4$, the simulation results of the co-evolution sensitivity for the conversion coefficient of the construction waste recycling industrial chain are shown in Fig. 4. 


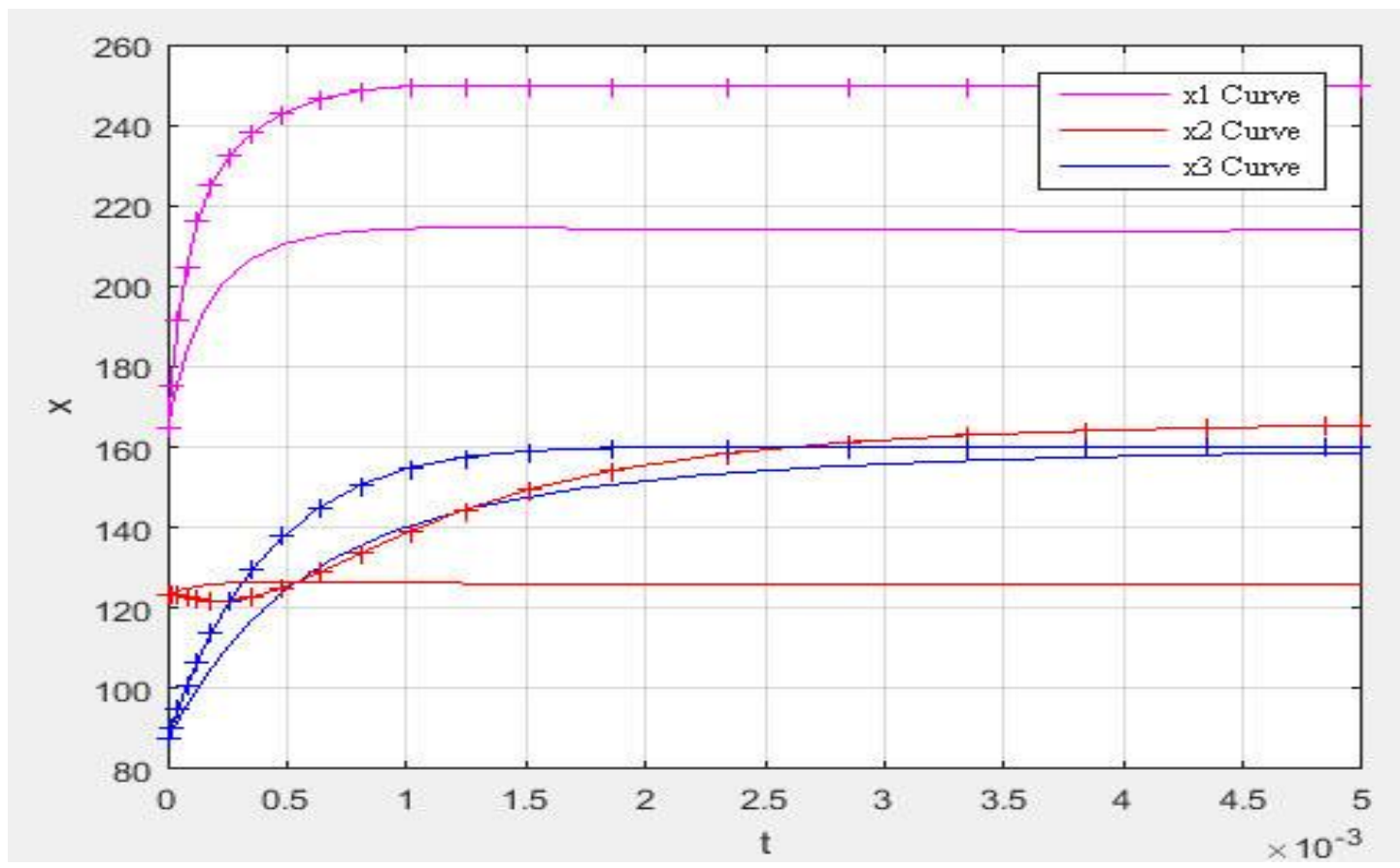

Fig. 4 Sensitivity analysis of the conversion coefficient of the construction waste recycling industrial chain meeting the conditions of Case 1

When the conversion coefficients are increased by $30 \%$, the conditions of Case 4 are met, and the analysis of Fig. 4 shows that the stable values of the output of recycled products from the construction waste recycling enterprises, the output of construction waste from the construction contractors, and the sales of recycled products by the recycled product dealers are $166.4,250$, and 160, respectively, which are consistent with the stable equilibrium point $A_{10}(166.4,250,160)$ in Case 4. In addition, the changes in evolution rates of the three parameters are evident: The evolution rate of the recycled product output of the construction waste recycling enterprises increases first and then decreases, and finally tends to be stable at 0 , whereas that of construction wastes from construction contractors and sales of recycled products by the recycled products dealers is accelerated. During this evolution process, it should be highlighted that the output of recycled products of construction waste recycling enterprises first decreases, then increases, and tends to be stable at 166.4 with strong fluctuation. When the conversion coefficients are reduced by $30 \%$, the parameters do not meet the condition for judging the stability of local equilibrium points.

\section{B. Co-evolution tendency and sensitivity analyses under conditions of Cases 4 and 10}

1) The tendency of co-evolution of the construction waste recycling industrial chain

The industrial chain of construction waste recycling is considered as the research object, and the initial output of enterprises, market demand $K$ of construction contractors on recycled products, and maximum production $D$ of construction waste of construction contractors are assigned in the co-evolution model of construction waste recycling industrial chain, $x_{10}=164.6$, $x_{20}=123.45, \quad x_{30}=87.5, \lambda=0.64$ (i.e., ) $K=160$ $D=250, \quad \beta_{1}=0.5, \beta_{2}=0.15, \quad \beta_{3}=0.2, \varepsilon_{1}=1.8$, $\varepsilon_{2}=1.1, \tau_{1}=1.6$, and $\tau_{2}=1.5$.

Based on the above data, the stability analysis of the local equalization points $A_{9}\left(\tau_{1} D, D, \lambda D\right)$, $A_{10}\left(\varepsilon_{2} \lambda D, D, \lambda D\right) \quad, \quad A_{11}\left(\varepsilon_{2} \lambda D, \varepsilon_{1} \varepsilon_{2} \lambda D, \lambda D\right) \quad, \quad$ and $A_{12}\left(\tau_{1} D, D, \tau_{1} \tau_{2} D\right)$ can be performed. The results show 
that if the conditions in Cases 4 and 10 are met, $\left\{\begin{array}{l}\beta_{1} \beta_{2} \beta_{3}\left(\varepsilon_{2} \lambda-\tau_{1}\right)\left(1-\varepsilon_{2} \tau_{2}\right)\left(1-\varepsilon_{1} \varepsilon_{2} \lambda\right)>0 \\ \beta_{2} \varepsilon_{2} \lambda\left(\varepsilon_{2} \lambda-\tau_{1}\right)+\beta_{1}\left(1-\varepsilon_{1} \varepsilon_{2} \lambda\right)+\beta_{3} \lambda^{2}\left(1-\varepsilon_{2} \tau_{2}\right)<0\end{array}\right.$ and $\left\{\begin{array}{l}-\beta_{1} \beta_{2} \beta_{3} \tau_{1}^{3} \tau_{2}\left(\tau_{2} \varepsilon_{2}-1\right)\left(\varepsilon_{1} \tau_{1}-1\right)\left(\lambda-\tau_{1} \tau_{2}\right)>0 \\ -\beta_{2} \tau_{1}^{2}\left(\tau_{2} \varepsilon_{2}-1\right)-\beta_{1}\left(\varepsilon_{1} \tau_{1}-1\right)-\beta_{3} \tau_{1} \tau_{2}\left(\lambda-\tau_{1} \tau_{2}\right)<0\end{array}\right.$, then $\tau_{1}>\varepsilon_{2} \lambda$ and $\varepsilon_{1} \varepsilon_{2} \lambda>1$. Therefore,
$A_{10}(176,250,160)$ is a stability point in the co-evolution process of the construction waste recycling industrial chain.

The simulation validation was performed in 0.005 as the simulation cycle. The simulation results of the coevolution of the industrial chain of construction waste recycling are shown in Fig. 5.

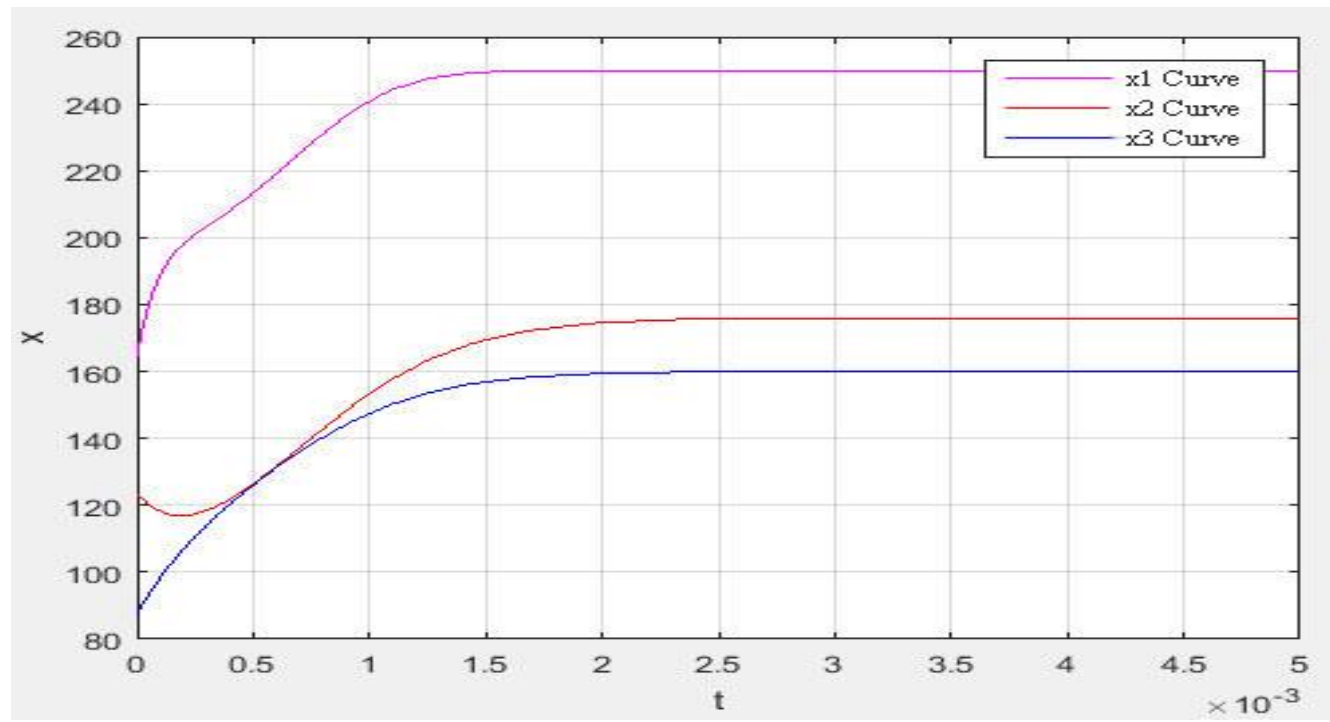

Fig. 5 Simulation of the co-evolution of the industrial chain of the construction waste recycling meeting the conditions of Case 4

The analysis of Fig. 5 shows that the output of recycled products of construction waste recycling enterprises initially decreases gradually from an initial output of 123.45 , and then increases to 176 with time. It reaches a stable state at $2.3 \times 10^{-3}$. The growth rate of the output of recycled products first increases and then decreases and tends to be stable at 0 ; The output of construction wastes of construction contractors gradually increases from an initial output of 164.6 to 250 , reaches a stable state at $1.5 \times 10^{-3}$, and then the growth rate of construction waste output gradually decreases and becomes stable at 0 ; The sales volume of recycled products of the recycled products dealers gradually increases from an initial output of 87.5 to 160 , and reaches a stable state at $2 \times 10^{-3}$. The growth rate of construction waste output gradually decreases and becomes stable at 0 . Thus, it is clear that the simulation results are in accordance with the analysis results.

2) Sensitivity analysis of the co-evolution of construction waste recycling industrial chain

Assuming that other conditions remain unchanged, that is $x_{10}=164.6, x_{20}=123.45, x_{30}=87.5, K=160$, $D=250, \quad \varepsilon_{1}=1.8, \varepsilon_{2}=1.1, \tau_{1}=1.6$, and $\tau_{2}=1.5$, and increasing and reducing by $30 \%$ the natural growth rates $\beta_{1}=0.5, \beta_{2}=0.15$, and $\beta_{3}=0.2$, the simulation results of the co-evolution sensitivity of the natural growth rate of the construction waste recycling industrial chain are shown in Fig. 6. 


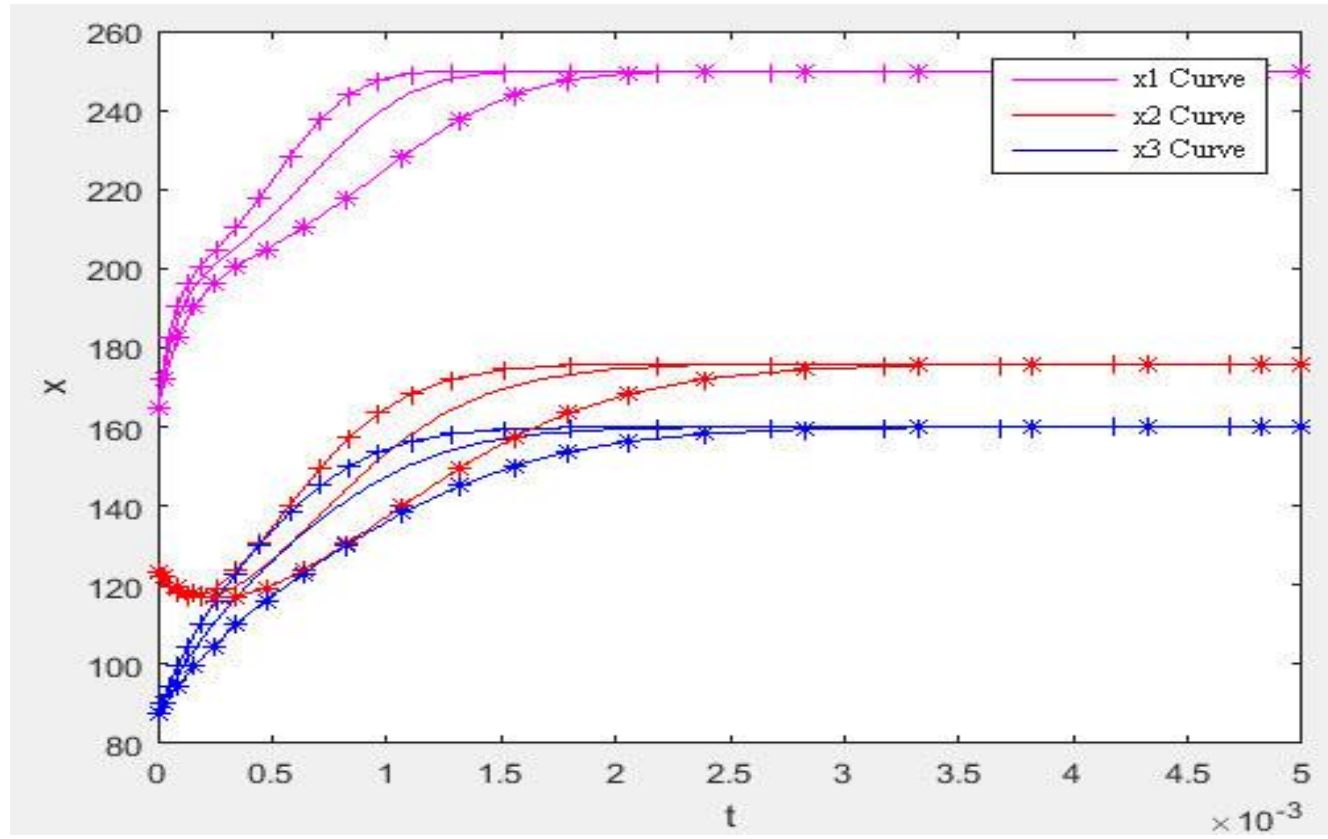

Fig. 6 Sensitivity analysis of the natural growth rate of the construction waste recycling industrial chain meeting the conditions of Case

Analysis of Fig. 6 shows that when the natural growth rates are increased by $30 \%$, the stable values of the output of recycled products from the construction waste recycling enterprises, the output of construction waste from the construction contractors and the sales of recycled products by the recycled products dealers are 176, 250 and 160, respectively, which are the same as the original stable values; The evolution rates of these three parameters are accelerated at the same time. When the natural growth rates are reduced by $30 \%$, the stable values are 176,250 , and 160 , respectively, which are the same as the original stable values; The evolution rates of the three parameters are decelerated at the same time. Thus, it is clear that whether the natural growth rates are increased or decreased by $30 \%$, the output curves are basically similar to the original values.

Assuming that other conditions remain unchanged, that is $x_{10}=164.6, x_{20}=123.45, x_{30}=87.5, K=160$, $D=250, \beta_{1}=0.5, \beta_{2}=0.15$, and $\beta_{3}=0.2$, and increasing and reducing by $30 \%$ the conversion coefficients $\varepsilon_{1}=1.8, \varepsilon_{2}=1.1, \tau_{1}=1.6$, and $\tau_{2}=1.5$, the simulation results of the co-evolution sensitivity of the conversion coefficient of the construction waste recycling industrial chain are shown in Fig. 7. 


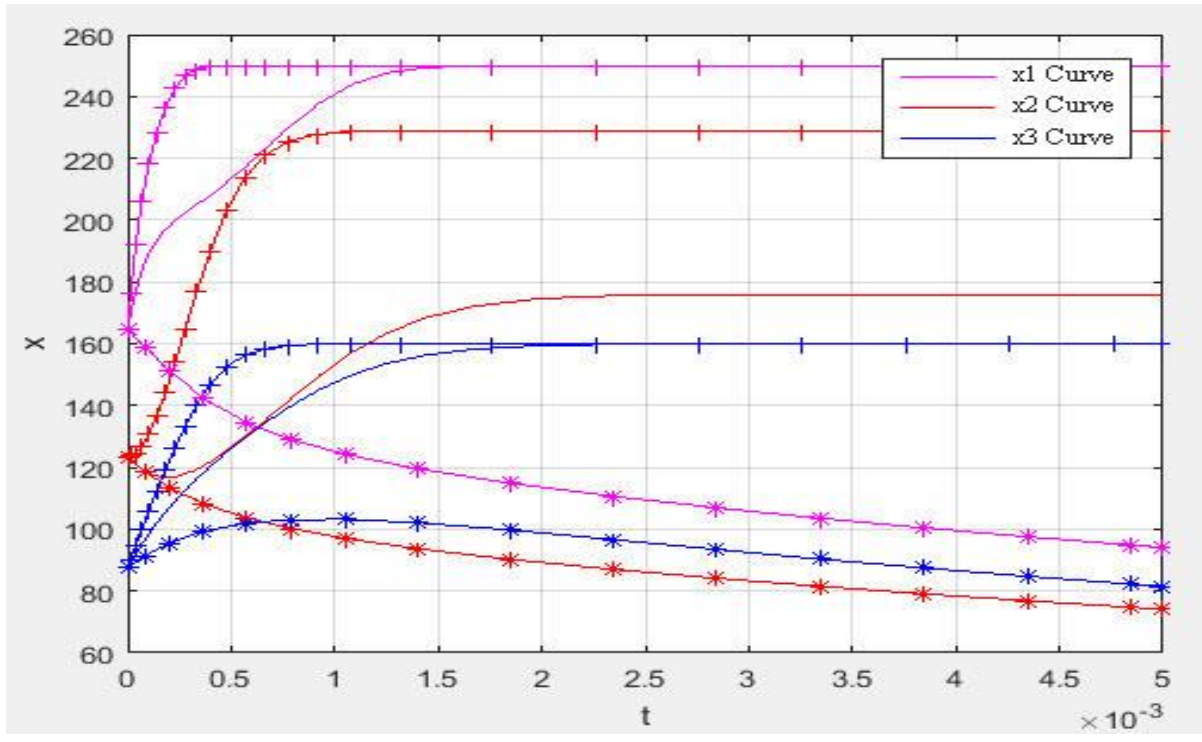

Fig. 7 Sensitivity analysis of the conversion coefficient of the construction waste recycling industrial chain meeting the conditions of

Case 4

When the conversion coefficients are increased by $30 \%$, the conditions of Case 4 are met, and the analysis of Fig. 7 shows that the stable values of the output of recycled products from the construction waste recycling enterprises, the output of construction waste from the construction contractors, and the sales of recycled products by the recycled products dealers are 228.8, 250, and 160, respectively, which are identical to the stable equilibrium point $\mathrm{A}_{10}(228.8,250,160)$ in Case 4 . In addition, the changes in the evolution rates of the three parameters are evident, accelerating and then reaching stability at $1 \times 10^{-3}$, $0.4 \times 10^{-3}$, and $0.8 \times 10^{-3}$, respectively, which are better than the original values of $2.3 \times 10^{-3}, 1.5 \times 10^{-3}$, and $2 \times$ $10^{-3}$. When the conversion coefficients are reduced by $30 \%$, the parameters do not meet the requirements of the local equilibrium stability judgment.

\section{Co-evolution tendency and sensitivity analyses under conditions of Case 7}

1) Analysis of the tendency of co-evolution of construction waste recycling industrial chain

The industrial chain of construction waste recycling is considered as the research object, and the initial output of enterprises, market demand $K$ of construction contractors on recycled products, and maximum production $D$ of construction waste of construction contractors are assigned in the co-evolution model of the construction waste recycling industrial chain, $x_{10}=164.6$, $x_{20}=123.45, x_{30}=87.5, \lambda=0.64$ (i.e., ) $K=160$ $D=250, \quad \beta_{1}=0.5, \quad \beta_{2}=0.15, \quad \beta_{3}=0.2, \quad \varepsilon_{1}=1.5$, $\varepsilon_{2}=1.1, \tau_{1}=0.7$, and $\tau_{2}=1.3$.

Based on the above data, the stability analysis of the local equalization points $A_{9}\left(\tau_{1} D, D, \lambda D\right)$, $A_{10}\left(\varepsilon_{2} \lambda D, D, \lambda D\right) \quad, \quad A_{11}\left(\varepsilon_{2} \lambda D, \varepsilon_{1} \varepsilon_{2} \lambda D, \lambda D\right) \quad, \quad$ and $A_{12}\left(\tau_{1} D, D, \tau_{1} \tau_{2} D\right)$ can be performed. The results show that if the conditions in Case 7 are met $\left\{\begin{array}{l}\beta_{1} \beta_{2} \beta_{3} \varepsilon_{1} \varepsilon_{2}{ }^{3} \lambda^{5}\left(\tau_{1} \varepsilon_{1}-1\right)\left(1-\varepsilon_{1} \varepsilon_{2} \lambda\right)\left(\tau_{2} \varepsilon_{2}-1\right)<0 \\ -\beta_{2} \varepsilon_{2}{ }^{2} \lambda^{2}\left(\tau_{1} \varepsilon_{1}-1\right)-\beta_{1} \varepsilon_{1} \varepsilon_{2} \lambda\left(1-\varepsilon_{1} \varepsilon_{2} \lambda\right)-\beta_{3} \lambda^{2}\left(\tau_{2} \varepsilon_{2}-1\right)<0\end{array}\right.$ , then $\tau_{1}<\varepsilon_{2} \lambda$ and $\tau_{1} \tau_{2}>\lambda$. Therefore, $A_{9}(175,250,160)$ is a stability point in the co-evolution process of the construction waste recycling industrial chain.

The simulation validation was performed in 0.100 as the simulation cycle. The simulation results of the coevolution of the industrial chain of construction waste recycling meeting the conditions of Case 7 are shown in Fig. 8. 


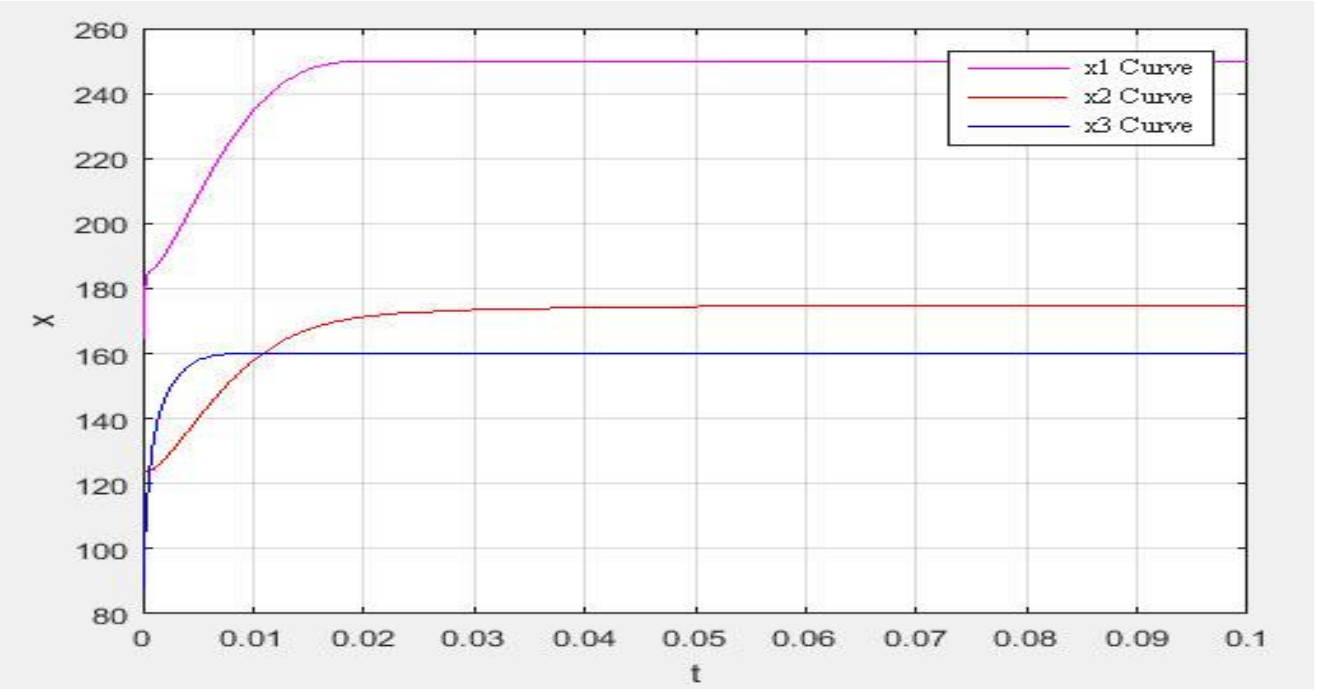

Fig. 8 Simulation of the co-evolution of the industrial chain of the construction waste recycling meeting the conditions of Case 7

The analysis of Fig. 8 shows that the output of recycled products of construction waste recycling enterprises gradually increases from the initial output of 123.45 to 174.9 , reaching a stable state at 0.05 , and the growth rate of this output gradually decreases and tends to be stable at 0 ; The output of construction wastes of construction contractors gradually increases from an initial output of 164.6 to 250 , reaching a stable state at 0.02 , and the growth rate of this output gradually decreases and becomes stable at 0 ; The sales volume of recycled products of the recycled products dealers gradually increases from an initial output of 87.5 to 160 , reaching a stable state at 0.01 , and the growth rate of this output gradually decreases and becomes stable at 0 . Thus, it is clear that the simulation results are in accordance with the analysis results.

2) Sensitivity analysis of the co-evolution of the construction waste recycling industrial chain

Assuming that other conditions remain unchanged, that is $x_{10}=164.6, x_{20}=123.45, x_{30}=87.5, K=160$, $D=250, \quad \varepsilon_{1}=1.5, \quad \varepsilon_{2}=1.1, \tau_{1}=0.7$, and $\tau_{2}=1.3$, and increasing and reducing by $30 \%$ the natural growth rates $\beta_{1}=0.5, \beta_{2}=0.15$, and $\beta_{3}=0.2$, the simulation results of the co-evolution sensitivity of the natural growth rate of the construction waste recycling industrial chain are shown in Fig. 9. 


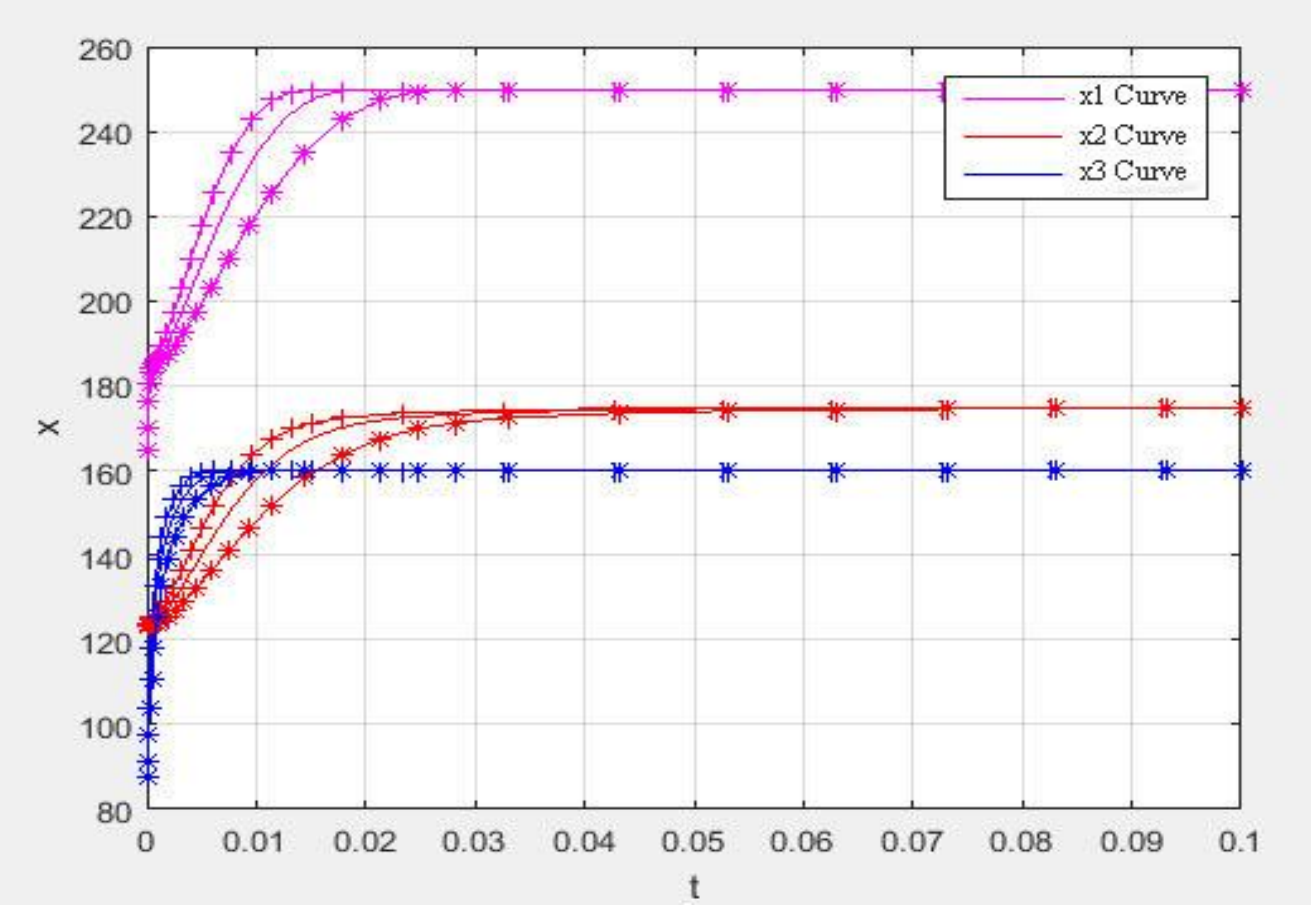

Fig. 9 Sensitivity analysis of the natural growth rate of the construction waste recycling industrial chain meeting the conditions of Case

Analysis of Fig. 9 shows that when the natural growth rates are increased by $30 \%$, the stable values of the output of recycled products from the construction waste recycling enterprises, the output of construction waste from the construction contractors and the sales of recycled products by the recycled products dealers are 174.9, 250 and 160, respectively, which are the same as the original stable values; The evolution rates of these three parameters are accelerated at the same time. When the natural growth rates are reduced by $30 \%$, the stable values of these outputs are $174.9,250$, and 160 , respectively, which are the same as the original stable values. The evolution rates of the three parameters are decelerated at the same time. Thus, it is clear that whether the natural growth rates are increased or decreased by $30 \%$, the output curves are basically similar to the original values.

Assuming that other conditions remain unchanged, that is $x_{10}=164.6, x_{20}=123.45, x_{30}=87.5, K=160$, $D=250, \beta_{1}=0.5, \beta_{2}=0.15$, and $\beta_{3}=0.2$, and increasing and reducing by $30 \%$ the conversion coefficients $\varepsilon_{1}=1.5, \varepsilon_{2}=1.1, \tau_{1}=0.7$, and $\tau_{2}=1.3$, the simulation results of the co-evolution sensitivity of the conversion coefficient of the construction waste recycling industrial chain are shown in Fig. 10. 


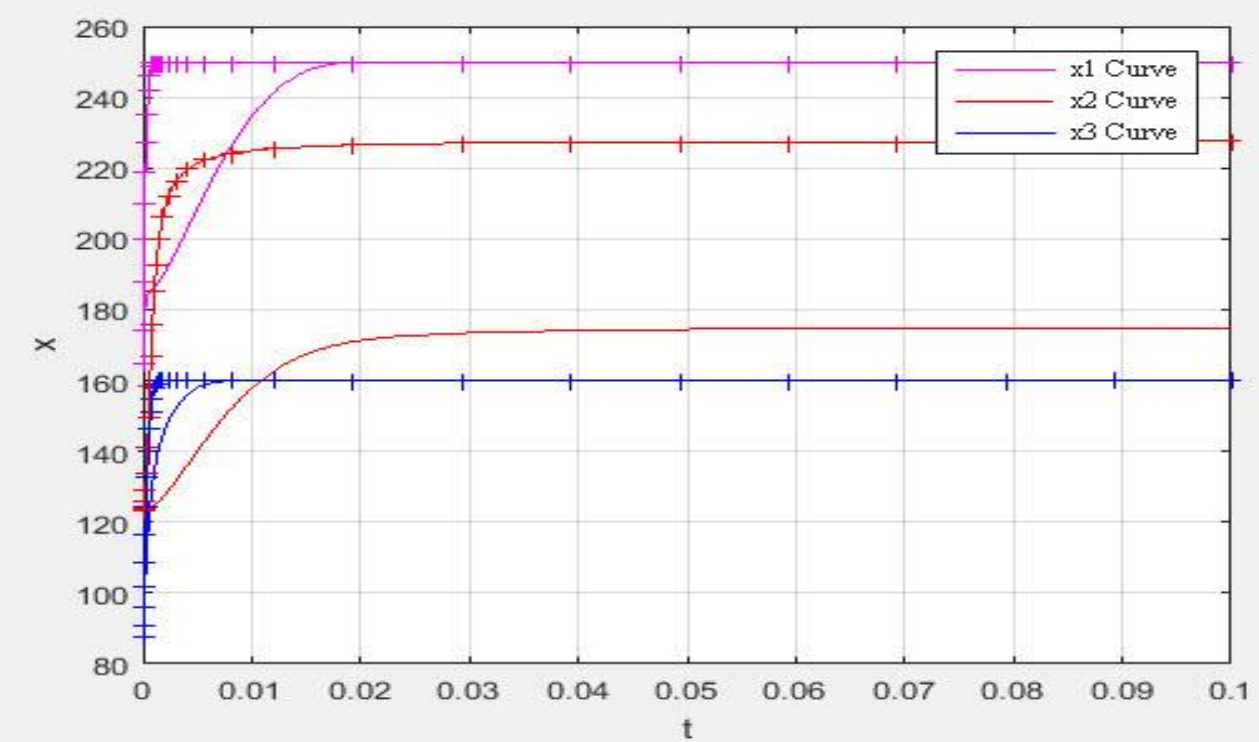

Fig. 10 Sensitivity analysis of the conversion coefficient of the construction waste recycling industrial chain meeting the conditions of

Case 7

When the conversion coefficients are increased by $30 \%$, the conditions of Case 7 are met, and the analysis of Fig. 10 shows that the stable values of the output of recycled products from the construction waste recycling enterprises, the output of construction waste from the construction contractors, and the sales of recycled products by the recycled products dealers are $174.9,250$, and 160 , respectively, which are basically consistent with the stable equilibrium point $\mathrm{A}_{10}(175,250,160)$ in Case 7. In addition, the changes in the evolution rates of the three parameters are evident, accelerating and then reaching stability at 0.03 , 0.001 , and 0.001 , respectively, which are better than the original values of $0.05,0.02$ and 0.01 . When the conversion coefficients are reduced by $30 \%$, the parameters do not meet the requirements of the local equilibrium stability judgment.

\section{Analysis and discussion}

According to the above analysis in Figs. 2, 5, and 8, the comparison between the validation results of the simulation of the co-evolution of the construction waste recycling industrial chain and the results of the stability analysis by the Jacobian matrix under the conditions of parameters meeting Cases 1, 4, 7, and 10 is shown in Table III.

Table III. Error analysis of stability analysis results and simulation verification results

\begin{tabular}{cccc}
\hline $\begin{array}{c}\text { Conditions of } \\
\text { parameters }\end{array}$ & $\begin{array}{c}\text { Stability analysis } \\
\text { results }\end{array}$ & $\begin{array}{c}\text { Simulation verification } \\
\text { results }\end{array}$ & Error rate \\
\hline Meeting Case 1 & $(128,217.6,160)$ & $(124.8,213.9,158.7)$ & $(2.5 \%, 1.7 \%, 0.8 \%)$ \\
$\begin{array}{c}\text { Meeting Cases } 4 \\
\text { and 10 }\end{array}$ & $(176,250,160)$ & $(176,250,160)$ & $(0 \%, 0 \%, 0 \%)$ \\
Meeting Case 7 & $(175,250,160)$ & $(174.9,250,160)$ & $(0.05 \%, 0 \%, 0 \%)$ \\
\hline
\end{tabular}

Table III shows that when the parameters meet the conditions of Case 1, the error rates of output of recycled products of construction waste recycling enterprises, output of construction wastes from construction contractors, and sales of recycled products of recycled products dealers are $2.5 \%, 1.7 \%$, and $0.8 \%$, respectively, by simulation verification results and stability analysis results, which meet the error range. When the parameters 
meet the conditions of Cases 4 and 10, the error rates of simulation verification results and stability analysis results are $0 \%, 0 \%$, and $0 \%$, respectively, which meet the error range. When the parameters meet the conditions of Case 7 , the error rates of the simulation verification results and stability analysis results are $0.05 \%, 0 \%$, and $0 \%$, respectively, which meet the error range.

According to the above analysis of Figs. 3, 4, 6, 7, 9, and 10 , it is clear that when parameters meet the conditions

\section{CASE SIMULATION}

After confirming the validity of the model, the coevolution of the construction waste recycling industrial chain in Guangzhou was simulated and forecasted. Based on the studies of Kloppers and Greeff the initial output of enterprises (construction waste recycling enterprises, recycled product dealers, and construction contractors), market demand $K$ of construction contractors on recycled products, and maximum production $D$ of construction waste (including waste from recycled products and traditional building materials as well as waste of Cases 1, 4, 7, and 10, the influence of the conversion coefficient is more sensitive than that of the natural growth rate on the industrial chain, which is in accordance with the research conclusions of Zhou [32].

Based on the above analysis, the simulation results of the co-evolution model of construction waste recycling industrial chain are in accordance with the stability analysis results, which verifies the reliability of the model and analysis [33] - [35].

such as residues) of construction contractors are reasonably predicted from 2020 to 2030: $x_{20}=1157.43$, $x_{10}=1543.24, x_{30}=820, \quad K=2360.6, \quad D=4551.6$ (in 10000 tons per year) [36].

The natural growth rate and other parameters between 2020 and 2030 were collected through a questionnaire survey. The parameters are listed in Table IV. The change in the simulation forecast curve and the numerical forecast of nodes in each year (year-end) about the co-evolution of construction waste recycling industrial chain in Guangzhou are shown in Fig. 11 and Table IV.

Table IV. Parameter values

\begin{tabular}{|c|c|c|c|c|c|c|c|}
\hline Parameters & $\beta_{1}$ & $\beta_{2}$ & $\beta_{3}$ & $\varepsilon_{1}$ & $\varepsilon_{2}$ & $\tau_{1}$ & $\tau_{2}$ \\
\hline Values & 0.55 & 0.17 & 0.28 & 0.64 & 1.45 & 1.72 & 0.83 \\
\hline \multicolumn{8}{|c|}{ Table V Node value forecast for each year (in 10000 tons/year) } \\
\hline & Year/variable & & $x_{1}$ & & $x_{2}$ & & $x_{3}$ \\
\hline & 2020 & & 1543.24 & & 1157.43 & & 820 \\
\hline & 2021 & & 1842.74 & & 1159.62 & & 938.43 \\
\hline & 2022 & & 1981.91 & & 1155.1 & & 1184.84 \\
\hline & 2023 & & 1985.63 & & 1155.21 & & 1528.03 \\
\hline & 2024 & & 2042.47 & & 1189.31 & & 1694.7 \\
\hline & 2025 & & 2447.99 & & 1426.19 & & 2015.53 \\
\hline & 2026 & & 3004.23 & & 1750.33 & & 2328.35 \\
\hline & 2027 & & 3328.12 & & 1935.64 & & 2360.6 \\
\hline & 2028 & & 3370.02 & & 1959.3 & & 2360.6 \\
\hline & 2029 & & 3369.99 & & 1959.3 & & 2360.6 \\
\hline & 2030 & & 3369.99 & & 1959.3 & & 2360.6 \\
\hline
\end{tabular}

Data from SBGP and GBS [37] [38] 


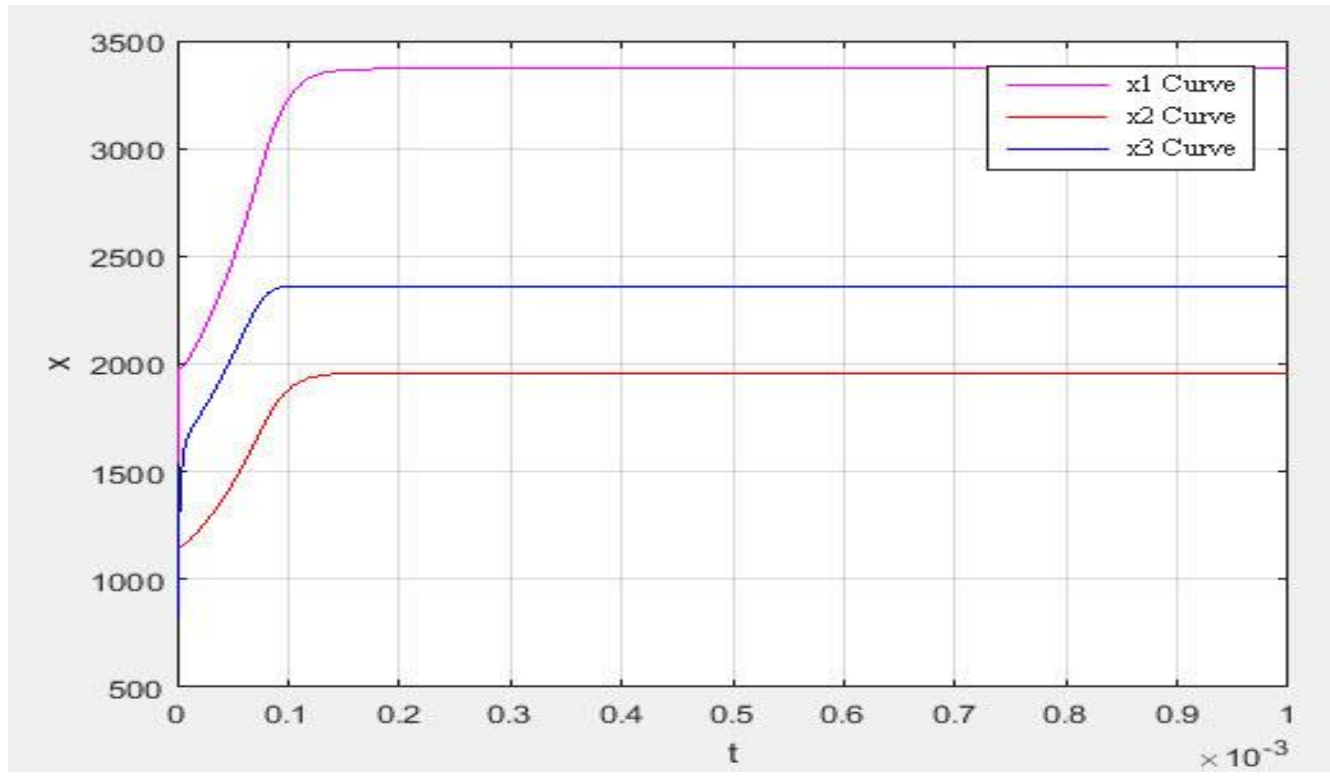

Fig. 11 Forecast of the co-evolution of the construction waste recycling industrial chain in Guangzhou City

The simulation results in Fig. 11 and Table V show that the output of recycled products of construction waste recycling enterprises reaches a stable state in 2028 , with an annual output of 19.593 million tons. The output of construction waste recycled by construction contractors reaches a stable state in 2028, with an annual handling capacity of 33.7 million tons, and the sales of recycled products of the recycled products dealers reaches a stable state in 2027, with annual sales of 23.606 million tons, indicating that the construction waste recycling industrial chain in Guangzhou will finally reach a stable state with a very high construction waste recycling rate, but lower than $100 \%$.

\section{CONCLUSIONS}

Aiming at the low utilization rate of construction waste resources in China, a Lotka-Volterra model of coevolution of construction waste recycling industry chain was built from the perspective of the construction waste recycling industry chain. The stability of the model was analyzed using the Jacobian matrix. The analysis results were verified by MATLAB numerical simulation. The simulation results indicated the following:

1) Regardless of the parameters, the error rate of the verification results and the stability analysis results from the simulation of output of recycled products of construction waste recycling enterprises, output of construction waste recycled by the construction contractors, and sales of recycled products of the recycled products dealers is less than $5 \%$, which proves that the coevolution model is reliable;

2) The output of recycled products of construction waste recycling enterprises, output of construction waste recycled by the construction contractors, and sales of recycled products of the recycled products dealers will reach their maximum. This indicates that the upstream and downstream enterprises in the entire construction waste recycling industrial chain will flourish, develop and progress together to realize industrial value added, and that they will constitute a stable product supply chain and maximize the total output value of the industrial chain;

3) The evolution rate of upstream and downstream enterprises in the construction waste recycling industrial chain is ultimately 0 , and the change rate is basically the same in the process of evolution, which indicates that there is a high product correlation between upstream and downstream enterprises in the construction waste recycling industrial chain;

4) Compared with the natural growth coefficient, the conversion coefficient has a higher influence and sensitivity on the evolution of construction waste recycling industrial chains. In the process of co-evolution of the construction waste recycling industrial chain, the 30\% increase in the natural growth rate and in the conversion 
factor of the industrial chain leads to a maximum increase of 0.2 and 19 times the rate of evolution of the industrial chain, respectively, resulting in a substantial increase in the synergistic efficiency.

In the process of co-evolution of the construction waste recycling industrial chain, only when the upstream and downstream enterprises of industrial chain work closely together to improve the conversion coefficient of enterprise output by means of information sharing, use of high-efficiency technology, and diversified processing of products to accelerate the co-evolution of the industrial chain and achieve a stable state, can the synergy effect of industrial chain finally be highlighted. As the propeller of the construction waste recycling industrial chain, the government should provide guidance, regulation, and incentive in the development and perfection, such as the formulation of standards for use of recycled products, government subsidy policies, government penalties, etc., and increase the market publicity to promote an increase in the market demand for recycled products.

In terms of practical significance, this study can guide the effective treatment of construction wastes on construction sites, help to improve the utilization rate of construction wastes as resources, help the government formulate incentive policies, and then improve the efficiency of policies to maximize the overall value of the industrial chain. In terms of theory, the related theories of construction waste management in China have been deepened in this study, as the basis for the government to formulate economic compensation policies and relevant laws and regulations in the process of resource management of construction waste, which can be used by the government to guide construction waste recycling and promote the development of the construction waste recycling industrial chain through market-oriented control and economic policies, to achieve the strategic goal of sustainable development.

There are many deficiencies in this study. For example, the construction waste recycling industry chain involves more stakeholders, and the related network structure is more complex, but only the main stakeholders are reflected in this study. Furthermore, the process of construction waste recycling is studied macroscopically from the perspective of industrial chains. In future studies, the impact of microindividual interactions in the construction waste recycling industrial chain can be explored from a micro perspective, in combination with self-organization and system complexity theories, to find its internal mechanism and evolution path.

\section{ACKNOWLEDGMENT}

The authors are grateful to all people who participated in the questionnaire survey and the illustrative example. They also sincerely thank the editors and the anonymous reviewers whose comments and suggestions improved and clarified this paper.

\section{References}

[1] J. K. Liu., Y. Q Yi, and X. T.Wang, "Exploring factors influencing construction waste reduction: A structural equation modeling approach," Journal of Cleaner Production, vol 276, pp. 123185, July 2020

[2] J. R. Li, Y. Yao, J. Zuo, and J. G. Li, "Key policies to the development of construction and demolition waste recycling industry in China," Waste Management, vol 108, pp. 137-143, May 2020

[3] S. O. Ajayi and L. O. Oyedele, "Policy imperatives for diverting construction waste from landfill: experts' recommendations for UK policy expansion," Journal of Cleaner Production, vol 147, pp. 57-65, Jan. 2017

[4] European Union, Construction and demolition waste (C\&DW)-2016. Available at http://ec.europa.eu/environment/waste/construction demolition.htm (accessed 25 April 2017).

[5] J. L. Galvez-Martos, D. Styles, H. Schoenberger, and B. Zeschmar-Lahl, "Construction and demolition waste best management practice in Europe," Resources, Conservation \& Recycling, vol 136, pp.166-178, Sept. 2018.

[6] Y. L. Wang, H. D. Guo, X. Wang, and K, Tao, "Research on social responsibility mechanism of industry chain participant on construction waste recycling," Construction Economy, vol 2, pp. 83-89, Feb. 2016.

[7] J. L. Vincent, J. Gan, and C. P. Cheng, "Formulation and analysis of dynamic supply chain of backfill in 
construction waste management using agent-based modeling," Advanced Engineering Informatics, vol 29, pp. 878-888, Oct. 2015.

[8] Y. Q Xiao, "Study on Recycling of Construction Waste from the Perspective of Industry Chain", Master of Science in Civil Engineering Thesis, Guangzhou University, Guangzhou, China, 2018.

[9] C. $\mathrm{Xu}$, "Research of the City Construction Waste Problem Based 0n System Dynamics," Master of Science in Civil Engineering Thesis, Beijing Forestry University, Beijing, China, 2015.

[10] H. Yang, J. Q. Xia, J. R. Thompson, and R. J. Flower, "Urban construction and demolition waste and landfill failure in Shenzhen, China," Waste Management, vol 63, pp. 393-396, May 2017.

[11] K. Murat, E. Gokhan, and T. Omer, "Evaluating environmental impacts of alternative construction waste management approaches using supply-chainlinked life-cycle analysis," Waste Management and Research, vol 32, pp. 500-508, May 2014.

[12] M. M. Hu, T. T. Liu, and S. Y. Shi, "Status and promoting advice for construction and demolition waste recycling industry: Based on analysis with the diamond model," Construction Economy, vol 4, pp. 74-79, Apr. 2016.

[13] Q. S. Gao and L. Xie, "The Study about key nodes and industrial development driving force of the C\&D waste resource utilization industry chain," Ecological

Economy, vol 6, pp. 137-141, Jun. 2014.

[14] G. Liu, Q. Z. Jian, and T. You, “Analysis on green building materials industry chain's evolutionary game based on circular economy," Science and Technology Management Research, vol 4, pp. 144-148, Apr. 2014.

[15] R. P. Côté, J. Lopez, S. Marche, G. M. Perron, and R. Wright, "Influences, practices and opportunities for environmental supply chain management in Nova Scotia SMEs," Journal of Cleaner Production, Vol 16, pp. 1561-1570, Oct. 2008.

[16] X. P. Li, D. Cai, and C. X. Guo, "Logistics network design of construction waste reduction system based on multi-dimensional perspective," System Engineering Theory and Practice, Vol 11, pp. 28422854, Nov. 2019.
[17] H. Y. Wu, J. Zuo, H. P. Yuan, G. Zillante, and J. Wang, "Cross-regional mobility of construction and demolition waste in Australia: An exploratory study," Resources, Conservation \& Recycling, Vol 156, pp. 104710, May 2020.

[18] H. El-Owaidy and A. Al-Thumairi, "On perturbation of the Lotka-Volterra model," Applied Mathematics Letters, Vol 4, pp. 557-560, Apr. 2009.

[19] H. J. Chen, X. J. Lou, and L. F. Wu, "Estimation the parameters of Lotka-Volterra model based on the grey direct modeling and its application," Mathematics in Practice and Theory, Vol 23, pp. 108-113, Nov. 2011.

[20] E. Odum, Fundamentals of ecology (3rd Ed.). W.B. Saunders Co., Philadelphia, 2017, pp. 54-60.

[21] T. T. Zhou and W. P. Wang, "Study on coordination of provincial industrial eco-economic system based on Lotka-Volterra model," Chinese Journal of Management Science, Vol 22(S1), pp. 240-246, Oct. 2014.

[22] H. J. Yang and J. H. Hu, "Yunnan Province based on the Lotka-Volterra model," Ecological Economy, Vol 5, pp. 60-65, May 2018.

[23] A. Szolnoki, M. Mobilia, L. L. Jiang, B. Szczesny, and M. Perc, "Cyclic dominance inevolutionary games: A review," Journal of the Royal Society Interface, Vol 11, pp. 20140735 , Nov. 2014.

[24] F. M. Lauro, M. Z. Demaere, S. Yau, M. V. Brown, and R. Cavicchioli, "An integrative study of a meromictic lake ecosystem in Antarctica," The Isme Journal, Vol 5, pp. 879-895, May 2011.

[25] J. H. Bao, X. R. Mao, G. Yin, et al., "Competitive Lotka-Volterra population dynamics with jumps," Nonlinear Analysis, Vol 17, pp. 6601-6616, Dec. 2011.

[26] A. R. J. Dainty and R. J. Brooke, “Towards improved construction waste minimization: A need for improved supply chain integration?" Struct Survey, Vol 1, pp. 20-29, Feb. 2004.

[27] K. Green and S. Randles, Industrial Ecology and Spaces of Innovation. Edward Elgar Publishing, 2006, pp. 114-130.

[28] H. A. Mohammed, G. Andrea, and A. Adolf, "Comparing linear and circular supply chains: A case study from the construction industry," International 
Journal of Production Economics, Vol 183, pp. 443457, Jan. 2017.

[29] N. Seror, S. Hareli, and B. A. Portnov, "Evaluating the effect of vehicle impoundment policy on illegal construction and demolition waste dumping: Israel as a case study," Waste Management, Vol 8, pp. 14361445, Aug. 2014.

[30] H. P. Yuan, A. R. Chini, Y. J. Lu, and L. Shen, "A dynamic model for assessing the effects of management strategies on the reduction of construction and demolition waste," Waste Management, Vol 3, pp. 520-530, Mar. 2012.

[31] W. Zhao, R. B. Leeftink, and V. S. Rotter, "Evaluation of economic feasibility for the recycling of construction and demolition waste in China-the case of Chongqing," Resources, Conservation \& Recycling, Vol 6, pp. 377-389, Jun. 2010.

[32] M. Zhou, "The research on the Synergetic Mechanism of housing industrialization from the perspective of industry chain, Master of Management Science and Engineering," Huaqiao University, Xiamen, China. 2016.

[33] P. T. Anh, D. T. N. Huy, and B. T. T. Loan, “Analysis of a Financial Model for Converting Industrial Waste

Yanqing Xiao received the B.S. degree from Shandong University of Science and Technology, Qingdao, China, in 2015, the M.S. degree from Guangzhou University, Guangzhou, China, in 2018. He is currently Consultant engineer of Guangzhou Municipal Construction Group Co., Ltd., Guangzhou, China. His research interests include Construction Waste Management, Construction Management and Economics.

Jingkuang Liu (Corresponding Author) received the B.S. degree from Yichun College, Jiangxi, China, in 2006, the M.S. degree from Guangzhou University, Guangzhou, China, in 2010, and Ph.D. degree from South China University of Technology, Guangzhou, China, in 2013. He is currently Associate Professor of Guangzhou University, Guangzhou, China. His research interests include Automation in Construction, Construction Waste Management, Construction Management and Economics.
Tires into Clean Energy for Environment Protection A Model in Developing Countries," WSEAS Transactions on Environment and Development, Vol 15, pp. 447-454, Apr. 2019.

[34] J. S. Yeomans, "Water Management using Populationbased Simulation-Optimization," WSEAS Transactions on Information Science and Applications, Vol 16, pp. 164-173, Apr. 2019.

[35] S. Kristiyana and A.Rinaldi, “Air Quality Monitoring System in Thingspeak-based Applications using Internet of Things (IOT)," WSEAS Transactions on Computer Research, Vol 8, pp. 34-38. Apr. 2020.

[36] P. H. Kloppers and J. C. Greeff. "Lotka-Volterra model parameter estimation using experiential data," Applied Mathematics and Computation, Vol 224, pp. 817-825, Nov. 2013.

[37] Statistics Bureau of Guangdong Province (SBGP), Statistical Yearbook of Guangdong Province, China Statistical Publishing House, Beijing, China, 2012, pp. 64-76.

[38] Guangzhou Bureau of Statistics (GBS), Statistical Yearbook of Guangzhou City, China Statistical Publishing House, Beijing, China, 2017, pp. 84-90.

\section{Author Contributions:}

Yanqing Xiao carried out the experiment and original draft preparation.

Jingkuang Liu did the study conceiving and methodology. Yongshi Pang was was responsible for review and editing.

\section{Sources of funding for research presented in a} scientific article or scientific article itself

Humanities and Social Sciences Projects of Ministry of Education (No.20YJCZH097), Guangdong Planning Office of Philosophy and Social Science (No.GD19CGL23).

\section{Creative Commons Attribution License 4.0 (Attribution 4.0 International, CC BY 4.0)}

This article is published under the terms of the Creative Commons Attribution License 4.0 https://creativecommons.org/licenses/by/4.0/deed.en_US 